Pacific

Journal of

Mathematics

AN APPROACH TO THE TANGENTIAL POISSON COHOMOLOGY BASED ON EXAMPLES IN DUALS OF LIE ALGEBRAS

Angela Gammella 


\title{
AN APPROACH TO THE TANGENTIAL POISSON COHOMOLOGY BASED ON EXAMPLES IN DUALS OF LIE ALGEBRAS
}

\author{
Angela Gammella
}

\begin{abstract}
In this paper, we are interested in the tangential Poisson cohomology (TP-cohomology) of regular Poisson manifolds, a cohomology which was first defined by Lichnerowicz using contravariant tensor fields. We show that for a regular Poisson manifold $M$, the TP-cohomology coincides with the leafwise de Rham (or Čech) cohomology of the symplectic foliation of $M$. Computing the spaces of such a cohomology leads actually to open and quite nontrivial problems. To get a better understanding of these difficulties, we study explicitly many examples coming from nilpotent and 3-dimensional (real) Lie algebras. For the latter, we compare the TP-cohomology and the usual Poisson cohomology (P-cohomology).
\end{abstract}

\section{Introduction and motivation.}

This work fits into the study of deformation quantization for the dual $\mathfrak{g}^{*}$ of a Lie algebra $\mathfrak{g}$, more exactly of star products on $\mathfrak{g}^{*}$ (or on some natural open subset $U$ of $\mathfrak{g}^{*}$ ) which restrict nicely to the coadjoint orbits contained in $\mathfrak{g}^{*}$ (or $U$ ). Such star products are called tangential and for a given Lie algebra $\mathfrak{g}$, they can notably be used to describe the harmonic analysis of the corresponding Lie group.

In general, tangential star products do not exist on the whole dual $\mathfrak{g}^{*}$ (see $[\mathbf{A C G}]$ or $[\mathbf{C G R}]$ ), nevertheless we know there always exists such a star product on the dense subset $\Omega$ of maximal dimensional coadjoint orbits in $\mathfrak{g}^{*}$ [Mas]. When studying all the possible classes of tangential star products on this set $\Omega$, we became interested in regular Poisson structures and especially in the TP-cohomology of regular Poisson manifolds. (Indeed tangential star products are governed by the TP-cohomology; for instance their classification is described by the second TP-cohomology space.) In this respect, it is worth mentioning Remarks 5 and 8 of the paper, which give an example of how our work applies to the theory of tangential star products.

With this motivation from deformation theory, we present here the result of our attempts to understand and clarify the TP-cohomology. We organize the paper as follows. 
In Section 2 below, we prove that, for a regular Poisson manifold, the TPcohomology is isomorphic to the leafwise de Rham (or Čech) cohomology of the symplectic foliation. Thus it depends only on that foliation and not on the symplectic structure along the leaves. This is a generalization of the fact that the P-cohomology of a symplectic manifold $M$ is just the de Rham cohomology of $M$. We recall also some classical results of foliation theory computing the TP-cohomology of some particular regular Poisson manifolds. We compare these results with a theorem from [Va2] describing the P-cohomology for some specific cases.

The remaining of the paper is devoted to examples arising from Lie algebras. Indeed, each Lie algebra $\mathfrak{g}$ gives rise to a natural regular Poisson manifold, namely the union $\Omega$ of all maximal dimensional coadjoint orbits in the dual space $\mathfrak{g}^{*}$.

In Section 3, we consider nilpotent Lie algebras $\mathfrak{g}$. For such Lie algebras, given a Jordan-Hölder basis $B, \Omega$ has a natural layering whose first layer, say $V_{B}$, is known as the generic (dense) open subset of $\mathfrak{g}^{*}$ associated to $B$ $([\mathbf{A C G}, \mathbf{V e r}])$. It is easy to see that the TP-cohomology of $V_{B}$ is trivial in degree superior to zero. We prove here that the same is true for the union $\cup_{B} V_{B}$, which is more canonical than $V_{B}$ since it does not depend on the choice of the basis $B$. However, $\cup_{B} V_{B}$ is sometimes strictly smaller than $\Omega$; this happens for instance in the case of the filiform Lie algebras. We will also see, by studying in details the case of the filiform Lie algebra $\mathfrak{g}_{4,1}$, that the TP-cohomology of $\Omega$ can be essentially larger than the TP-cohomology of $\cup_{B} V_{B}$. Such an example shows that the TP-cohomology of regular Poisson manifolds (and more generally the leafwise de Rham cohomology of foliations) can be huge even if the leaves are cohomologically trivial.

Later, in Section 4, we consider an arbitrary 3-dimensional regular Poisson manifold $M$ and we perform the inductive computations of [Va2] to describe the P-cohomology spaces of $M$. This enables us to observe the influence of the TP-cohomology on the P-cohomology: The TP-cohomology spaces appear naturally in the decomposition of the P-cohomology spaces (see Proposition 6). Then, we examine the TP-cohomology and the P-cohomology of the regular Poisson manifold $\Omega$ arising from any 3-dimensional Lie algebra. Some of these Lie algebras can be directly treated with the help of Section 2, the others will require more attention. We conclude with some general remarks.

\section{Regular Poisson manifolds and foliation theory.}

2.1. Basic definitions. A Poisson manifold is a $C^{\infty}$ manifold $M$ equipped with a Poisson bracket $\{$,$\} i.e., a bilinear skew-symmetric operation on$ 
$C^{\infty}(M)$ with values in $C^{\infty}(M)$, satisfying the Leibniz rule:

$$
\{f, g h\}=\{f, g\} h+g\{f, h\} \quad \forall f, g, h \in C^{\infty}(M)
$$

and the Jacobi identity:

$$
\{\{f, g\}, h\}+\{\{g, h\}, f\}+\{\{h, f\}, g\}=0 \quad \forall f, g, h \in C^{\infty}(M) .
$$

For any manifold $M$, we denote by $\mathcal{V}^{*}(M)$ the graded space of skewsymmetric contravariant tensor fields and by $\Omega^{*}(M)$ the graded space of forms on $M$.

Let $M$ be a Poisson manifold. Since the Poisson bracket is skew-symmetric and satisfies the Leibniz rule, there exists a unique tensor field $\Lambda$ in $\mathcal{V}^{2}(M)$ such that

$$
\{f, g\}=\Lambda(d f, d g) \quad \forall f, g \in C^{\infty}(M) .
$$

This tensor field is usually called the Poisson bivector of $M$.

To express the Jacobi identity in terms of $\Lambda$, we recall that the commutator bracket of vector fields extends to the Schouten bracket, uniquely defined on $\mathcal{V}^{*}(M)$ by the relations:

(i) $[P, Q]=-(-1)^{(p-1)(q-1)}[Q, P] \quad \forall P \in \mathcal{V}^{p}(M), \forall Q \in \mathcal{V}^{q}(M)$.

(ii) For $P$ in $\mathcal{V}^{p}(M),[P,$.$] is a derivation of degree p-1$.

The Schouten bracket satisfies the graded Jacobi identity:

$$
[P,[Q, R]]=[[P, Q], R]+(-1)^{(p-1)(q-1)}[Q,[P, R]],
$$

for $P$ in $\mathcal{V}^{p}(M), Q$ in $\mathcal{V}^{q}(M), R$ in $\mathcal{V}^{*}(M)$, and thus defines a graded Lie algebra structure on $\mathcal{V}^{*}(M)$ with the shifted grading: $\operatorname{deg}(S)=s-1$ if $S$ belongs to $\mathcal{V}^{s}(M)$. One can then check [Li1, Wei] that the bracket on $C^{\infty}(M)$ given by $\Lambda$ satisfies the Jacobi identity if and only if $[\Lambda, \Lambda]=0$ holds.

In the sequel, we shall denote by $(M, \Lambda)$ our Poisson manifold. If $f$ is a $C^{\infty}$ function on $M$, we call Hamitonian vector field of $f$ the vector field corresponding to the derivation $\{f,$.$\} . With (M, \Lambda)$ is associated a bundle map:

$$
\begin{aligned}
\#: T^{*} M & \longrightarrow T M \\
\alpha & \longmapsto \alpha^{\#}
\end{aligned}
$$

defined by

$$
\alpha^{\#}(\beta)=\Lambda(\alpha, \beta)
$$

for any $\alpha, \beta$ in $T_{x}^{*} M$. Finally, the rank of $M$ at a point $x$ is by definition the rank of the linear mapping $\#_{x}: T_{x}^{*} M \rightarrow T_{x} M$. If it is constant, $M$ is said to be regular. In particular, if it is everywhere equal to the dimension of $M$, \# is an isomorphism and $M$ is a symplectic manifold whose symplectic structure $\omega$ is given by $\omega=\#^{-1}(\Lambda)$. 
We are now ready to define the Poisson cohomology of the Poisson manifold $(M, \Lambda)$.

Definition 1. Let $\sigma: \mathcal{V}^{*}(M) \rightarrow \mathcal{V}^{*+1}(M)$ be the operator given by

$$
\sigma=[\Lambda, .] .
$$

Due to the graded Jacobi identity for [, ], $\sigma$ is a coboundary operator (i.e., $\left.\sigma^{2}=0\right)$. The complex $\left(\mathcal{V}^{*}(M), \sigma\right)$ is called the Poisson complex of $M$ and the corresponding cohomology $H_{\Lambda}^{*}(M)$ is the P-cohomology of $M$.

See $[$ Hue] for an algebraic definition of the P-cohomology and $[\mathbf{A P P}$, CW] for some more recent results about the P-cohomology.

The interpretation of the first few P-cohomology spaces is well-known. Indeed, $H_{\Lambda}^{0}(M)$ is the space $I(M)$ of Casimir functions over $M$ i.e., those whose Hamiltonian vector fields are trivial; $H_{\Lambda}^{1}(M)$ consists of infinitesimal Poisson automorphisms of $M$ (Poisson vector fields) modulo inner automorphisms (Hamiltonian vector fields); $H_{\Lambda}^{2}(M)$ classifies (modulo the trivial deformations) the formal deformations of the Poisson structure $\Lambda$ (with the form $\left.\Lambda+t \alpha_{1}+t^{2} \alpha_{2}+\ldots\right)$ and finally $H_{\Lambda}^{3}(M)$ houses the obstructions to extend a formal deformation from one step (in powers of $t$ ) to the next.

Let us just recall that the equivalence classes of star products on $(M, \Lambda)$ are in one to one correspondence with the equivalence classes of formal deformations of $\Lambda$ (see [Kon] for more details).

Note also that the Poisson bracket gives rise to a bracket $\{$,$\} on \Omega^{1}(M)$, which is the unique extension of the bracket given by $\{d f, d g\}=d\{f, g\}$ such that

$$
\{\alpha, f \beta\}=f\{\alpha, \beta\}+\left(\alpha^{\#} f\right) \beta \quad \forall f \in C^{\infty}(M) \quad \forall \alpha, \beta \in \Omega^{1}(M) .
$$

This bracket is defined by

$$
\{\alpha, \beta\}=L_{\alpha \#}(\beta)-L_{\beta \#}(\alpha)-d(\Lambda(\alpha, \beta)) \quad \forall \alpha, \beta \in \Omega^{1}(M)
$$

and one can prove (see [Va2, p. 44])

$$
\begin{aligned}
\sigma Q\left(\alpha_{0}, \ldots, \alpha_{k}\right)= & \sum_{i=0}^{k}(-1)^{i} \alpha_{i}^{\#}\left(Q\left(\alpha_{0}, \ldots, \hat{\alpha_{i}}, \ldots, \alpha_{k}\right)\right) \\
& +\sum_{i<j}(-1)^{i+j} Q\left(\left\{\alpha_{i}, \alpha_{j}\right\}, \alpha_{0}, \ldots, \hat{\alpha_{i}}, \ldots, \hat{\alpha_{j}}, \ldots, \alpha_{k}\right)
\end{aligned}
$$

where $Q$ is in $\mathcal{V}^{k}(M)$ and the $\alpha_{i}$ are 1 -forms on $M$.

The latter expression can be used ([Va2]) to see that the natural extension \# of \# to forms:

$$
\widetilde{\#} \lambda\left(\alpha_{0}, \ldots, \alpha_{q-1}\right)=(-1)^{q} \lambda\left(\alpha_{0}^{\#}, \ldots, \alpha_{q-1}^{\#}\right)
$$


intertwines $\sigma$ and the de Rham differential $d$ and thus induces a natural homomorphism from $H_{D R}^{*}(M)$ to $H_{\Lambda}^{*}(M)$. This homomorphism is trivially an isomorphism in the symplectic case (see also [Kos] or [Li1]).

Some preparatory material related to a foliation is now needed. Let $(M, \mathcal{F})$ be an arbitrary foliated manifold and denote by $T \mathcal{F}$ the tangent bundle of $\mathcal{F}$. As in $[\mathbf{D H}]$ or $[\mathbf{L i 2}]$, one can choose a transversal distribution $\nu \mathcal{F}$ such that

$$
T M=T \mathcal{F} \oplus \nu \mathcal{F} \quad \text { and } \quad T^{*} M=T^{*} \mathcal{F} \oplus \nu^{*} \mathcal{F} .
$$

These decompositions induce a bigrading of the space $\mathcal{V}^{*}(M)$ of contravariant tensor fields and of the space $\Omega^{*}(M)$ of forms on $M$, namely

$$
\mathcal{V}^{*}(M)=\underset{p, q}{\oplus} \mathcal{V}_{p, q}(M) \quad \text { and } \quad \Omega^{*}(M)=\bigoplus_{p, q} \Omega_{p, q}(M)
$$

where $\mathcal{V}_{p, q}(M)$ (resp. $\Omega_{p, q}(M)$ ) denotes the space of sections of the bundle $\wedge^{q}(T \mathcal{F}) \otimes \wedge^{p}(\nu \mathcal{F})\left(\right.$ resp. $\left.\wedge^{q}\left(T^{*} \mathcal{F}\right) \otimes \wedge^{p}\left(\nu^{*} \mathcal{F}\right)\right)$.

Elements of $\mathcal{V}_{p, q}(M)$ and $\Omega_{p, q}(M)$ are said to be of type $(p, q)$. Moreover, an operator will be homogeneous of type $(a, b)$ if it sends elements of type $(p, q)$ to elements of type $(p+a, q+b)$. We recall that the de Rham differential $d$ can be decomposed into $d=d^{\prime}+d^{\prime \prime}+d_{2,-1}$ where $d^{\prime}$ is of type $(1,0), d^{\prime \prime}$ denotes the leafwise de Rham differential of the foliation $\mathcal{F}$ and is of type $(0,1)$, and $d_{2,-1}$ is of type $(2,-1)$.

Assume now that $(M, \Lambda)$ is a regular Poisson manifold and denote by $\mathcal{F}$ the symplectic foliation of $M$. As above, one can choose a transversal distribution $\nu \mathcal{F}$ for $M$. It was shown in [Va2] that with respect to a given choice of $\nu \mathcal{F}$, the coboundary operator $\sigma$, introduced in Definition 1, has a well-defined decomposition $\sigma=\sigma^{\prime}+\sigma^{\prime \prime}$ where $\sigma^{\prime}$ is of type $(-1,2)$ and $\sigma^{\prime \prime}$ is of type $(0,1)$.

On the other hand, Lichnerowicz has shown in $[\mathbf{L i 2}]$ that one gets a consistent theory by restricting the P-cohomology complex $\left(\mathcal{V}^{*}(M), \sigma\right)$ to tangential multivector fields. The resulting cohomology is known as the TP-cohomology of the regular Poisson manifold $(M, \Lambda)$. In fact, the same cohomology can be defined by using the transversal distribution $\nu \mathcal{F}$ and the types of the tensor fields. Indeed, we have:

Definition 2. The TP-cohomology complex of the regular Poisson manifold $(M, \Lambda)$ is $\underset{q}{\oplus_{0, q}} \mathcal{V}_{0, q}(M)$ with the coboundary operator $\sigma^{\prime \prime}$ and

$$
H_{\Lambda, \tan }^{q}(M)=\frac{\operatorname{Ker}\left(\sigma^{\prime \prime}: \mathcal{V}_{0, q}(M) \rightarrow \mathcal{V}_{0, q+1}(M)\right)}{\operatorname{Im}\left(\sigma^{\prime \prime}: \mathcal{V}_{0, q-1}(M) \rightarrow \mathcal{V}_{0, q}(M)\right)}
$$

is the qth TP-cohomology space of $(M, \Lambda)$.

It is clear that $H_{\Lambda, \tan }^{0}(M)=H_{\Lambda}^{0}(M)$. Moreover, as mentioned in the introduction, the TP-cohomology plays an important role in the theory of 
tangential star products. Indeed, the derivations of a given tangential star product on $M$, modulo inner derivations, are parameterized by sequences of elements in $H_{\Lambda, \tan }^{1}(M)$; similarly equivalences of tangential star products on $M$ are classified at each step by $H_{\Lambda, \tan }^{2}(M)$ and finally the obstructions to construct such a star product are localized in $H_{\Lambda, \tan }^{3}(M)$ (this last point could be omitted since a tangential deformation always exists on $M$ [Mas]).

2.2. Leafwise de Rham cohomology. In this paragraph, we want to prove that the TP-cohomology of a regular Poisson manifold $(M, \Lambda)$ is isomorphic to the leafwise de Rham cohomology of the symplectic foliation and therefore does not depend on the symplectic structure along the leaves.

To this end, we consider first the general case of a foliated manifold $(M, \mathcal{F})$. For all $p$, we denote by $\Phi^{p}(\mathcal{F})$ the sheaf of (germs of) projectable $p$-forms (i.e., those induced by forms on the space of leaves). In particular, $\Phi^{0}(\mathcal{F})$ is the sheaf of the germs of functions on $M$ that are constant along the leaves of $\mathcal{F}$ (see $[\mathbf{V a 2}]$ ). Let us fix a transversal distribution $\nu \mathcal{F}$ and consider the sheaf cohomology of $\Phi^{p}(\mathcal{F})$, that is

$$
H^{q}\left(M, \Phi^{p}(\mathcal{F})\right)=\frac{\operatorname{Ker}\left(d^{\prime \prime}: \Omega_{p, q}(M) \rightarrow \Omega_{p, q+1}(M)\right)}{\operatorname{Im}\left(d^{\prime \prime}: \Omega_{p, q-1}(M) \rightarrow \Omega_{p, q}(M)\right)} .
$$

A change of $\nu \mathcal{F}$ leads to an isomorphism in the corresponding cohomology spaces. To see this, let us introduce more notations. Let $N \mathcal{F}$ be the normal bundle of $\mathcal{F}$, that is $N \mathcal{F}=T M / T \mathcal{F}$. Denote by $\Omega_{[p]}^{q}(\mathcal{F})$ the space of sections of the bundle $\wedge^{q}\left(T^{*} \mathcal{F}\right) \otimes \wedge^{p}\left(N^{*} \mathcal{F}\right)$. The elements of $\Omega_{[p]}^{0}(\mathcal{F})$ are usually called normal forms and those of $\Omega_{[p]}^{q}(\mathcal{F})$ are the tangential $q$-forms with values in the normal $p$-forms. The Lie algebra of tangential vector fields acts naturally (by Lie derivative) on the normal forms and the leafwise de Rham differential $d_{\mathcal{F}}$ acts on $\Omega_{[p]}^{q}(\mathcal{F})$ in the usual way:

$$
\begin{aligned}
& d_{\mathcal{F}}\left(\omega_{x}\right)\left(X_{0}, \ldots, X_{q}\right) \\
& =\sum_{i=0}^{q}(-1)^{i} L_{X_{i}}\left(\omega_{x}\left(X_{0}, \ldots, \hat{X}_{i}, \ldots, X_{q}\right)\right) \\
& \quad+\sum_{i<j}(-1)^{i+j} \omega_{x}\left(\left[X_{i}, X_{j}\right], X_{0}, \ldots, \hat{X}_{i}, \ldots, \hat{X}_{j}, \ldots, X_{q}\right),
\end{aligned}
$$

if $x$ is in $M, \omega$ in $\Omega_{[p]}^{q}(\mathcal{F})$ and the $X_{i}$ in $T_{x} \mathcal{F}$. Since $d_{\mathcal{F}}^{2}=0$, we obtain a com$\operatorname{plex}\left(\underset{q}{\oplus} \Omega_{[p]}^{q}(\mathcal{F}), d_{\mathcal{F}}\right)$ whose cohomology is denoted hereafter by $H_{[p]}^{*}(\mathcal{F})$.

With these notations, we can prove: 
Lemma 1. For all $p$, the complexes $\left(\underset{q}{\oplus} \Omega_{p, q}(M), d^{\prime \prime}\right)$ and $\left(\underset{q}{\oplus} \Omega_{[p]}^{q}(\mathcal{F}), d_{\mathcal{F}}\right)$ are isomorphic. In particular, $H^{*}\left(M, \Phi^{p}(\mathcal{F})\right)$ and $H_{[p]}^{*}(\mathcal{F})$ coincide and are independent of the choice of $\nu \mathcal{F}$.

Proof. For each $x$ in $M$, let $\psi_{x}: N_{x} \mathcal{F} \rightarrow \nu_{x} \mathcal{F}$ be the natural isomorphism of vector spaces. That is, if $Y$ is in $T_{x} M$, then $\psi_{x}(\widetilde{Y})=\pi(Y)$ where $\widetilde{Y}$ denotes the class of $Y$ in $N_{x} \mathcal{F}$ and $\pi$ is the projection $\pi: T M \rightarrow \nu \mathcal{F}$.

The mapping $f: \Omega_{p, q}(M) \rightarrow \Omega_{[p]}^{q}(M)$ given by

$$
f\left(\omega_{x}\right)\left(X_{1}, \ldots, X_{q}\right)\left(\widetilde{Y_{1}}, \ldots, \widetilde{Y_{p}}\right)=\omega_{x}\left(X_{1}, \ldots, X_{q}, \psi_{x}\left(\widetilde{Y_{1}}\right), \ldots, \psi_{x}\left(\widetilde{Y_{p}}\right)\right),
$$

here $\omega$ is in $\Omega_{p, q}(M)$, the $X_{i}$ in $T_{x} \mathcal{F}$, and the $Y_{j}$ in $T_{x} M$, is clearly bijective. In addition, we have

$$
\begin{aligned}
& d_{\mathcal{F}} f\left(\omega_{x}\right)\left(X_{0}, \ldots, X_{q}\right)\left(\widetilde{Y_{1}}, \ldots, \widetilde{Y_{p}}\right) \\
& =\sum_{i=0}^{q}(-1)^{i} X_{i}\left(f\left(\omega_{x}\right)\left(X_{0}, \ldots, \hat{X}_{i}, \ldots, X_{q}\right)\left(\widetilde{Y_{1}}, \ldots, \widetilde{Y_{p}}\right)\right) \\
& \quad-\sum_{i=0}^{q} \sum_{l=1}^{p}(-1)^{i+l-1} f\left(\omega_{x}\right)\left(X_{0}, \ldots, \hat{X}_{i}, \ldots, X_{q}\right)\left(\left[\widetilde{X_{i}, Y_{l}}\right], \widetilde{Y_{1}}, \ldots, \hat{Y}_{l}, \ldots, \widetilde{Y_{p}}\right) \\
& +\sum_{0 \leq i<j \leq q}(-1)^{i+j} f\left(\omega_{x}\right)\left(\left[X_{i}, X_{j}\right], X_{0}, \ldots, \hat{X}_{i}, \ldots, \hat{X}_{j}, \ldots X_{q}\right)\left(\widetilde{Y_{1}}, \ldots, \widetilde{Y_{p}}\right) \\
& =f\left(d^{\prime \prime} \omega_{x}\right)\left(X_{0}, \ldots, X_{q}\right)\left(\widetilde{Y_{1}}, \ldots, \widetilde{Y_{p}}\right) .
\end{aligned}
$$

Thus, $d_{\mathcal{F}} \circ f=f \circ d^{\prime \prime}$. This ends the proof.

Remark that $H^{q}\left(M, \Phi^{0}(\mathcal{F})\right)\left(\right.$ or $\left.H_{[0]}^{q}(\mathcal{F})\right)$ is nothing else but the leafwise de Rham cohomology of the foliation $\mathcal{F}$.

Let us now restrict ourselves to the case of a regular Poisson manifold $(M, \Lambda)$. Recall that $I(M)$ denotes the space of Casimir functions over $M$, in other words the space of smooth functions on $M$ that are constant along the leaves of the symplectic foliation. Then we have:

Theorem 1. Let $(M, \Lambda)$ be a regular Poisson manifold, $\mathcal{F}$ the symplectic foliation of $M$ and $\nu \mathcal{F}$ a transversal distribution for $M$. Then $\left(\underset{q}{\oplus} \mathcal{V}_{0, q}(M), \sigma^{\prime \prime}\right)$ and $\left(\underset{q}{\oplus} \Omega_{0, q}(M), d^{\prime \prime}\right)$ are isomorphic as complexes of $I(M)$-modules. In particular, for all $q, H_{\Lambda, \tan }^{q}(M)$ and $H^{q}\left(M, \Phi^{0}(\mathcal{F})\right)$ are isomorphic $I(M)$ modules.

Proof. It is not difficult to prove that the natural extension \# of \# realizes an $I(M)$-modules isomorphism between $\Omega_{0, q}(M)$ and $\mathcal{V}_{0, q}(M)$. This isomorphism satisfies $\sigma^{\prime \prime} \circ \widetilde{\#}=-\widetilde{\#} \circ d^{\prime \prime}$. The result follows. 
2.3. Ćech cohomology. Let $(M, \mathcal{F})$ be a foliated manifold. We shall say that a locally finite covering $\mathcal{U}=\left(U_{i}\right)$ of $M$ is a good covering if for all $q>0$, all $k$ and all $i_{1}, \ldots, i_{k}$,

$$
H^{q}\left(U_{i_{1}, \ldots, i_{k}}, \Phi^{0}\left(\mathcal{F}_{\mid U_{i_{1}, \ldots, i_{k}}}\right)\right)=\{0\},
$$

where $U_{i_{1}, \ldots, i_{k}}=U_{i_{1}} \cap \ldots \cap U_{i_{k}}$ and $\mathcal{F}_{\mid U_{i_{1}, \ldots, i_{k}}}$ denotes the foliation induced by $\mathcal{F}$ in $U_{i_{1}, \ldots, i_{k}}$ (i.e., if the leaves of $\mathcal{F}$ are noted $\mathcal{L}_{\alpha}$, the leaves of $\mathcal{F}_{\mid U_{i_{1}, \ldots, i_{k}}}$ are the connected components of the intersections $\left.\mathcal{L}_{\alpha} \cap U_{i_{1}, \ldots, i_{k}}\right)$.

We recall that, for each foliated manifold $(M, \mathcal{F})$, there exists affine connections on $M$, which are torsion free and adapted to $\mathcal{F}$ in the sense of [Li2]. Let us now prove the existence of good coverings.

Lemma 2. Let $(M, \mathcal{F})$ be a foliated manifold and $\Gamma$ an affine connection on $M$, which is torsion free and adapted to $\mathcal{F}$. Then, every atlas $\left\{U_{i}, \varphi_{i}\right\}$ on $M$ of distinguished charts has an open refinement $\left\{V_{l}, \psi_{l}\right\}$ such that:

(1) Each $V_{l}$ has compact closure.

(2) $\left(V_{l}\right)$ is locally finite and is a good covering of $M$.

Proof. By taking an open refinement if necessary, we may assume that $\left(U_{i}\right)$ is locally finite and that each $U_{i}$ has compact closure. Let $\left(U_{i}^{\prime}\right)$ be an open refinement of $\left(U_{i}\right)$ (with the same index set) such that $\overline{U_{i}^{\prime}} \subset U_{i}$ for all $i$, and note $\varphi_{i}^{\prime}=\varphi_{\left.i\right|_{U_{i}^{\prime}}}$. For each $x$ in $M$, let $W_{x}$ be a normal neighborhood of $x$, which is small enough to satisfy the following properties:

- for each point $a$ in $W_{x}$, there exists a normal neighborhood $N_{a}$ of 0 in $T_{a} M$ such that $\exp : N_{a} \rightarrow W_{x}$ is a diffeomorphism;

- $W_{x}$ is geodesically convex;

- $W_{x}$ is contained in some $U_{i}^{\prime}$.

Note $\varphi_{x}=\varphi_{\left.i\right|_{W x}}^{\prime}$ and, for each $k$, set

$$
B_{k}=\left\{\left(W_{x}, \varphi_{x}\right): W_{x} \cap \overline{U_{k}^{\prime}} \neq \emptyset\right\}
$$

Since $\overline{U_{k}^{\prime}}$ is compact, there exists a finite subfamily $B_{k}^{\prime}$ of $B_{k}$, which covers $\overline{U_{k}^{\prime}}$. Then the family $\mathcal{B}=\cup_{k} B_{k}^{\prime}$ is an open refinement $\left\{V_{l}, \psi_{l}\right\}$ of $\left\{U_{i}, \varphi_{i}\right\}$, which satisfies (1) and (2). In fact, it is clear by construction that each $V_{l}$ has compact closure and that $\left(V_{l}\right)$ is locally finite. To show that $\left(V_{l}\right)$ is also a good covering of $M$, we shall now prove that each $V_{l}$ has geodesically convex plaques. Let $y$ be in some $V_{l}$ and denote by $P_{y}$ the plaque of $\mathcal{F}_{\left.\right|_{V_{l}}}$ containing $y$. Take two points $a$ and $b$ in $P_{y}$. By assumption, there exists a normal neighborhood $N_{a}$ of 0 in $T_{a} M$ such that exp : $N_{a} \rightarrow V_{l}$ is a diffeomorphism. 
a) First, we shall prove that $\exp \left(N_{a} \cap T_{a} \mathcal{F}\right)=P_{a}$. Let $Y$ be in $N_{a} \cap T_{a} \mathcal{F}$. Denote by $\tau$ the geodesic of $V_{l}$ with the initial condition $(a, Y)$ :

$$
\tau(0)=a \quad \text { and } \quad \dot{\tau}(0)=\tau_{*_{0}}\left(\frac{d}{d t}\right)=Y .
$$

This curve $\tau$ is at least defined for $0 \leq t \leq 1$. We express it in the distinguished chart $\left(V_{l}, \psi_{l}\right)$ as follows

$$
x(t):=\psi_{l}(\tau(t))=\left(x^{i}(t), x^{u}(t)\right)
$$

where $i, j \ldots=1, \ldots, r$ (resp. $u, v, \ldots=1, \ldots, s)$ denote the tangential (resp. transverse) indexes. Since $\tau$ is a geodesic, it satisfies

$$
\begin{cases}\frac{d^{2} x^{i}}{d t^{2}}=-\sum_{1 \leq J, K \leq r+s} \Gamma_{J K}^{i}(x) \frac{d x^{J}}{d t} \frac{d x^{K}}{d t} \quad \text { for } \quad i=1, \ldots, r \\ \frac{d^{2} x^{u}}{d t^{2}}=-\sum_{1 \leq J, K \leq r+s} \Gamma_{J K}^{u}(x) \frac{d x^{J}}{d t} \frac{d x^{K}}{d t} \quad \text { for } \quad u=1, \ldots, s .\end{cases}
$$

Moreover, since $\Gamma$ is torsion free and adapted to $\mathcal{F}$, we have

$$
\Gamma_{i A}^{u}=\Gamma_{A i}^{u}=0 \quad \forall 1 \leq i \leq r, 1 \leq u \leq s, 1 \leq A \leq r+s .
$$

It follows that

$$
\frac{d^{2} x^{u}}{d t^{2}}=-\sum_{1 \leq v, w \leq s} \Gamma_{v w}^{u}(x) \frac{d x^{v}}{d t} \frac{d x^{w}}{d t} \quad \text { for } \quad u=1, \ldots, s .
$$

Let us introduce the notation

$$
f(t)=\left(x^{i}(t)\right)_{1 \leq i \leq r}, \quad g(t)=\left(x^{u}(t)\right)_{1 \leq u \leq s} .
$$

Then, the above system can be reduced to two ordinary differential equations of the form

$$
\begin{aligned}
& f^{\prime \prime}(t)=F\left(f^{\prime}(t), f(t), g^{\prime}(t), g(t)\right) \\
& g^{\prime \prime}(t)=G\left(g^{\prime}(t), g(t), f(t)\right) .
\end{aligned}
$$

Since $\dot{\tau}(0)=Y$ is in $T_{a} \mathcal{F}$, we shall have $g^{\prime}(0)=0$. Now, for fixed $f$ and with the initial conditions $g(0)$ and $g^{\prime}(0)=0,(2)$ has a unique solution namely $g=c s t=g(0)$. Let us denote by $f_{0}(t)=\left(a^{i}(t)\right)_{1 \leq i \leq r}$ the unique solution of (1) when $g=$ cst and with $f(0)$ and $f^{\prime}(0)$ as initial conditions. Then, we have $\left(x^{i}(t), x^{u}(t)\right)=\left(a^{i}(t), x^{u}(0)\right)$ for all $0 \leq t \leq 1$. In particular, $\tau(1)=\exp (Y)$ belongs to $P_{a}$. We get thus the inclusion $\exp \left(N_{a} \cap T_{a} \mathcal{F}\right) \subset P_{a}$. The equality comes from the fact that $\exp \left(N_{a} \cap T_{a} \mathcal{F}\right)$ is both open and closed in $P_{a}$, and that $P_{a}$ is connected.

b) Now, let $\gamma$ be the unique minimizing geodesic of $V_{l}$, joining $a$ and $b$. We may write

$$
\gamma:[0,1] \rightarrow V_{l}, \quad \gamma(t)=\exp (t X)
$$


where $\gamma(0)=a, \gamma(1)=b$ and $X$ is in $N_{a}$. Since $\gamma(1)=b=\exp (X)$ is in $P_{a}\left(=P_{y}\right)$ and using the equality proved in a), we see that $X$ is in fact in $N_{a} \cap T_{a} \mathcal{F}$. Thus, $\gamma$ lies entirely in $P_{y}$.

We have proved that $V_{l}$ has geodesically convex (hence contractible) plaques. It is of course the same for every finite intersection $V_{l_{1}, \ldots, l_{k}}$. Therefore, each foliation $\mathcal{F}_{\left.\right|_{V_{l_{1}, \ldots, l_{k}}}}$ is a product foliation by contractible leaves. Following a result of [Va2], we shall mention just in the next paragraph (Theorem 3), this means that $H^{q}\left(V_{l_{1}, \ldots, l_{k}}, \Phi^{0}\left(\mathcal{F}_{\left.\right|_{l_{l_{1}}, \ldots, l_{k}}}\right)\right)=\{0\}$ for all $q>0$. Lemma 2 is proved.

Now, for any sheaf $A$ on $M$ (in particular for $\Phi^{0}(\mathcal{F})$ ), we shall denote by $\check{C}^{k}(\mathcal{U}, A)$ the space of $k$-Čech cochains of $A$ with respect to a covering $\mathcal{U}$ of $M$, by $\check{\delta}$ the $\check{C}$ ech coboundary and $\check{\mathrm{H}}^{*}(\mathcal{U}, A)$ the cohomology corresponding to the Cech complex $\left(\check{C}^{*}(\mathcal{U}, A), \check{\delta}\right)$.

The purpose of the following proposition is to prove that the leafwise de Rham cohomology of a foliation $\mathcal{F}$ coincides with the Čech cohomology of the sheaf $\Phi^{0}(\mathcal{F})$. This can be convenient to calculate the TP-cohomology (see later in Section 3).

Proposition 1. Let $(M, \mathcal{F})$ be a foliated manifold and $\nu \mathcal{F}$ a transversal distribution for $M$. Let also $\mathcal{U}=\left(U_{i}\right)$ be a good covering of $M$ and $\left(h_{i}\right)$ a partition of unity subordinate to $\mathcal{U}$. For a $k$-Cech cocycle $c$, let $\omega_{c}$ be the $d^{\prime \prime}$-closed $k$-form on $M$ defined by

$$
\omega_{c \mid U_{i}}=(-1)^{\frac{k(k+1)}{2}} \sum_{i_{1}, \ldots, i_{k}} c_{i_{1}, \ldots, i_{k}, i} d^{\prime \prime} h_{i_{1}} \wedge \ldots d^{\prime \prime} h_{i_{k}} .
$$

Then, the homomorphism $\varphi: \check{H}^{k}\left(\mathcal{U}, \Phi^{0}(\mathcal{F})\right) \rightarrow H^{k}\left(M, \Phi^{0}(\mathcal{F})\right)$ mapping the cohomology class $[c]$ to the cohomology class $\left[\omega_{c}\right]$ is an isomorphism for all $k$.

Proof. The result can be proved in the same way as Brylinski did in [Bry] to show that the Cech cohomology of the constant sheaf $\mathbb{R}_{M}$ on any manifold $M$ coincides with the de Rham cohomology of $M$. The key point of the proof is to consider the Cech double complex $K^{* *}=\check{C}^{*}\left(\mathcal{U}, \Omega_{0, *}(M)\right)$ where underlining denote sheaves of germs. The total cohomology of $K^{* *}$, also called Čech hypercohomology, is by definition the cohomology corresponding to the complex $\left(K^{*}, D\right)$ where $K^{n}=\underset{p+q=n}{\bigoplus} K^{p, q}$ and $D=\check{\delta}+(-1)^{p} d^{\prime \prime}$ in degree $(p, q)$. It is known ([Bry, p. 28]) that the natural spectral sequences associated to $K^{*}$ lead to a canonical isomorphism between $\check{H}^{k}\left(\mathcal{U}, \Phi^{0}(\mathcal{F})\right)$ and $H^{k}\left(M, \Phi^{0}(\mathcal{F})\right)$. To prove this isomorphism is just $\varphi$, one needs first to see $c$ and $\omega_{c}$ as elements of $\check{C}^{k}\left(\mathcal{U}, \Omega_{0,0}(M)\right)$ and $\check{C}^{0}\left(\mathcal{U}, \Omega_{0, k}(M)\right)$ respectively.




other from the point of view of hypercohomology. The proposition follows directly.

2.4. Review of some classical results. We begin this paragraph by mentioning two well-known results of foliation theory. These results, which can be found in $[\mathbf{D H}]$ and $[\mathbf{V a 2}]$ respectively, provide the computation of the TP-cohomology in some particular cases.

Theorem 2. Let $(M, \mathcal{F})$ be a foliated manifold and $r$ some integer. Assume that the foliation $\mathcal{F}$ is given by a submersion $\Pi: M \rightarrow B$ ( $B$ being a Hausdorff manifold), and that any leaf $L$ of $\mathcal{F}$ is connected and satisfies $H_{D R}^{q}(L)=\{0\}$ for all $0<q \leq r$. Then,

$$
H^{q}\left(M, \Phi^{0}(\mathcal{F})\right)= \begin{cases}C^{\infty}(B) & \text { if } q=0 \\ \{0\} & \text { if } 0<q \leq r .\end{cases}
$$

Theorem 3. Let $L$ and $R$ be two smooth manifolds and $\mathcal{F}$ the foliation of $M=L \times R$ by the leaves $L \times\{x\}$ where $x$ is in $R$. Assume that $L$ has finite Betti numbers. Then,

$$
H^{q}\left(M, \Phi^{p}(\mathcal{F})\right)=H_{D R}^{q}(L) \otimes \Omega^{p}(R) .
$$

Next, in Theorem 4, we recall an important result from [Va1, Va2]. This result will be used in Section 4 when we shall compute the P-cohomology spaces associated to 3-dimensional Lie algebras.

Theorem 4. Let $M=S \times R$ be a regular Poisson manifold whose regular Poisson structure $\Lambda$ is transversally constant with respect to the transversal distribution $\nu \mathcal{F}=T R$ (i.e., the symplectic foliation $\mathcal{F}$ of $M=S \times R$ is defined by a fixed symplectic structure of $S$ ). Suppose that $S$ has finite Betti numbers. Then,

$$
H_{\Lambda}^{q}(M) \simeq \underset{0 \leq k \leq q}{\oplus} H_{D R}^{k}(S) \otimes \Omega^{q-k}(R) .
$$

Remark 1. One can use Theorem 4 to show that, with constrast to the TPcohomology, the P-cohomology not only depends on the symplectic foliation but also on the symplectic structure along the leaves. Indeed, let $M$ be $\mathbb{S}^{2} \times \mathbb{R}_{+}^{*}$ and denote by $\omega$ the standard symplectic structure on the unit sphere $\mathbb{S}^{2}$. If $M$ is endowed with the regular Poisson structure defined by the same symplectic structure $\omega$ on each leaf, then the P-cohomology of $M$ is given by Theorem 4 . But, if the same manifold $M$ is viewed as $\mathfrak{s u}(2)^{*} \backslash\{0\}$ with its usual linear Poisson structure, then each leaf $\mathbb{S}^{2} \times\{t\}\left(t \in \mathbb{R}_{+}^{*}\right)$ has a different symplectic structure, namely $t \omega$, and Theorem 4 is no more valid for $M$ (we will see the actual computation of $\mathfrak{s u}(2)^{*} \backslash\{0\}$ in $\S 4.7$ ).

Apart from the specific cases of Theorems 2 and 3, the task of computing the TP-cohomology still remains unsolved. To better understand the TP-cohomology of general regular Poisson manifolds and to make some 
comparison between the TP-cohomology and the P-cohomology, we devote the next two sections to a large number of explicit computations related to Lie algebras. More precisely, the regular Poisson manifolds we shall consider in the rest of the paper are Poisson submanifolds of the union $\Omega$ of all maximal dimensional coadjoint orbits in the dual of a given Lie algebra. That makes sense since the dual $\mathfrak{g}^{*}$ of any Lie algebra $\mathfrak{g}$ can be endowed with a natural Poisson structure, the well-known Lie Poisson structure [Wei]; the leaves of the symplectic foliation of $\mathfrak{g}^{*}$ being exactly the coadjoint orbits.

\section{The nilpotent case.}

Suppose that $\mathfrak{g}$ is an $m$-dimensional nilpotent Lie algebra. Denote by $\mathfrak{g}^{*}$ the dual space of $\mathfrak{g}$ and by $G$ the connected and simply connected Lie group with Lie algebra $\mathfrak{g}$. Let $\mathfrak{g}_{0} \subset \mathfrak{g}_{1} \subset \ldots \subset \mathfrak{g}_{m}=\mathfrak{g}$ be a flag of $\mathfrak{g}\left(\operatorname{dim} \mathfrak{g}_{i}=i\right)$ such that $\left[\mathfrak{g}, \mathfrak{g}_{i}\right] \subseteq \mathfrak{g}_{i-1}$ for all $i$ in $\{1, \ldots, m\}$. Let also $B=\left(X_{1}, \ldots, X_{m}\right)$ be a Jordan-Hölder basis adapted to $\left(\mathfrak{g}_{i}\right)_{i}$ that is $\mathfrak{g}_{i}=\mathbb{R} X_{1} \oplus \ldots \oplus \mathbb{R} X_{i}$ for all $i$. Then, $\mathfrak{g}^{*}$ (or $\Omega$ ) has a natural layering which can be summarized as follows (see also [ACG, Bon, Puk, Ver]).

For $\mu$ in $\mathfrak{g}^{*}$, we define the set of indexes $J_{\mu}=\left\{j: X_{j} \notin \mathfrak{g}_{j-1}+\mathfrak{g}_{\mu}\right\}$, where $\mathfrak{g}_{\mu}=\{X \in \mathfrak{g}: \forall Y \in \mathfrak{g},\langle\mu,[X, Y]\rangle=0\}$. If $J_{\mu}=\left\{j_{1}<\ldots<j_{2 r}\right\}$, we shall have $\mathfrak{g}=\mathfrak{g}_{\mu} \oplus \mathbb{R} X_{j_{1}} \ldots \oplus \mathbb{R} X_{j_{2 r}}$. Let $\Delta=\left\{J_{\mu}, \mu \in \mathfrak{g}^{*}\right\}$. For $e$ in $\Delta$, we define the following layer

$$
\Omega_{B}^{e}=\left\{\mu \in \mathfrak{g}^{*}: J_{\mu}=e\right\} .
$$

By construction, each layer is a $G$-invariant subset of $\mathfrak{g}^{*}$ and $\mathfrak{g}^{*}$ (resp. $\Omega$ ) is a disjoint finite union of layers $\mathfrak{g}^{*}=\cup_{e \in \Delta} \Omega_{B}^{e}\left(\right.$ resp. $\Omega=\cup_{e \in \Delta}\left(\Omega_{B}^{e} \cap \Omega\right)$ ). Note that all the orbits contained in a given layer have the same dimension ( $\operatorname{card} e$ ).

Now, let $\Omega_{B}^{e}$ be an arbitrary layer of $\mathfrak{g}^{*}$ and assume that the orbits contained in $\Omega_{B}^{e}$ are $2 r$-dimensional. Then, there exists on $\mathfrak{g}^{*}$

(i) $m-2 r$ polynomial functions $z_{1}, \ldots, z_{m-2 r}$

(ii) $2 r$ rational functions $p_{1}, \ldots, p_{r}, q_{1}, \ldots, q_{r}$ which are regular on $\Omega_{B}^{e}$ such that:

- the polynomial functions $z_{1}, \ldots, z_{m-2 r}$ separate the orbits contained in $\Omega_{B}^{e}$;

- for each orbit $O$ contained in $\Omega_{B}^{e}$, there is a diffeomorphism (a global Darboux chart) $\varphi: O \rightarrow \mathbb{R}^{2 r}$ of symplectic manifolds defined by the functions $p_{i}, q_{j}$.

The first layer, noted $V_{B}$, has additional properties: It is a Zariski dense open subset of $\mathfrak{g}^{*}$, it contains only orbits of maximal dimension $2 d$ and the polynomial functions $z_{1}, \ldots, z_{m-2 d}$ separating the orbits of $V_{B}$ are $G$ invariant. Moreover, if we identify the symmetric algebra $S(\mathfrak{g})$ of $\mathfrak{g}$ with the space of polynomial functions on $\mathfrak{g}^{*}$ and denote by $S(\mathfrak{g})^{G}$ the subring 
of $S(\mathfrak{g})$ of the $G$-invariant polynomial functions, then the quotient field of $S(\mathfrak{g})^{G}$ coincides exactly with the field $\mathbb{R}\left(z_{1}, \ldots, z_{m-2 d}\right)$ of rational functions in the $z_{k}$ variables. The open set $V_{B}$ is usually called the generic set associated to the basis $B$, the orbits contained in $V_{B}$ are the generic orbits and the corresponding polynomial functions $z_{1}, \ldots, z_{m-2 d}$ are the generic invariants. Since the symplectic foliation of $V_{B}$ is a product foliation whose leaves are contractible, it follows immediately from Theorem 3 that the TPcohomology of $V_{B}$ is trivial in degree superior to zero. The next proposition claims it is even possible to get rid of the choice of the basis $B$.

Proposition 2. Let $\mathfrak{g}$ be a nilpotent Lie algebra. Consider the union $\cup_{B} V_{B}$ of all possible generic sets associated to Jordan-Hölder bases $B$ of $\mathfrak{g}$ and denote by $\Lambda$ its regular Poisson structure. Then,

$$
H_{\Lambda, \tan }^{0}\left(\cup_{B} V_{B}\right)=I\left(\cup_{B} V_{B}\right) \quad \text { and } \quad H_{\Lambda, \tan }^{q}\left(\cup_{B} V_{B}\right)=\{0\} \quad \forall q>0 .
$$

Proof. We observe first that $\Pi: \cup_{B} V_{B} \rightarrow\left(\cup_{B} V_{B}\right) / G$ is a locally trivial fibration thus a submersion. Now, since the orbits contained in $\cup_{B} V_{B}$ are connected and cohomologically trivial, the result directly comes from Theorem 2 (Section 2).

Remark 2. It is known that $V_{B}$ is in general strictly included in the set $\Omega$ of all maximal dimensional coadjoint orbits (see [SG]). Unfortunately, $\cup_{B} V_{B}$ can also be strictly smaller than $\Omega$. For instance, in the case of the filiform Lie algebras (defined in $[\mathbf{C G}]$ or $[\mathbf{G K}]$ ), all the $V_{B}$ coincide and are distinct from $\Omega$.

Now, the following result is very convenient and quite efficient for many examples.

Proposition 3. Let $\mathfrak{g}$ be an $m$-dimensional nilpotent Lie algebra, $\mathfrak{g}^{*}$ the dual space of $\mathfrak{g}$ and $G$ the connected and simply connected Lie group with Lie algebra $\mathfrak{g}$. Denote by $\Omega$ the union of all the coadjoint orbits of maximal dimension $(2 d)$. Still denote by $B=\left(X_{i}\right)$ a Jordan-Hölder basis of $\mathfrak{g}$, by $V_{B}$ the generic set associated to the basis $B$ and by $z_{1}, \ldots, z_{m-2 d}$ the generic invariants separating the orbits of $V_{B}$. If $\widetilde{\Omega}$ is any open subset of $\Omega$ such that the polynomial functions $z_{1}, \ldots, z_{m-2 d}$ separate the orbits contained in $\widetilde{\Omega}$ and that the vectors $d z_{1}(\mu), \ldots, d z_{m-2 d}(\mu)$ are linearly independent for all $\mu$ in $\widetilde{\Omega}$, then the TP-cohomology of $\widetilde{\Omega}$ (endowed with its linear Poisson structure $\Lambda$ ) is given by

$$
H_{\Lambda, \tan }^{0}(\widetilde{\Omega})=I(\widetilde{\Omega}) \quad \text { and } \quad H_{\Lambda, \tan }^{q}(\widetilde{\Omega})=\{0\} \quad \forall q>0 .
$$


Proof. Let us consider the smooth mapping

$$
\begin{aligned}
f: \widetilde{\Omega} \times \widetilde{\Omega} & \longrightarrow \mathbb{R}^{m-2 d} \\
(\mu, \eta) & \longmapsto\left(z_{1}(\mu)-z_{1}(\eta), \ldots, z_{m-2 d}(\mu)-z_{m-2 d}(\eta)\right) .
\end{aligned}
$$

Note that $\Sigma=f^{-1}(0)$ is not empty (it contains the diagonal set $\Delta=$ $\{(\mu, \mu): \mu \in \widetilde{\Omega}\})$. Moreover, for all $(\mu, \eta)$ in $\Sigma$, the rank of the linear mapping $f_{*(\mu, \eta)}$ is, by assumption, equal to $m-2 d$. As a result, $\Sigma$ is a closed submanifold of $\widetilde{\Omega} \times \widetilde{\Omega}$. This exactly means (see [Die, p. 58]) that the space of leaves $\widetilde{\Omega} / G$ is Hausdorff and that the canonical projection $\Pi: \widetilde{\Omega} \rightarrow \widetilde{\Omega} / G$ is a submersion. The result is thus again a straightforward consequence of Theorem 2.

Remark 3. If some $\widetilde{\Omega}$ satisfies the conditions of Proposition 3 for a particular Jordan-Hölder basis, it satisfies these conditions for any Jordan-Hölder basis. Indeed, since the generic invariants $z_{1}, \ldots, z_{m-2 d}$ associated to any basis $B$ generate the quotient field of $S(\mathfrak{g})^{G}$, the fact that the coadjoint orbits contained in $\widetilde{\Omega}$ are separated or not by the polynomial functions $z_{1}, \ldots, z_{m-2 d}$, and therefore Proposition 3, do not depend on the choice of the basis $B$.

As one can see by studying the examples of Pedersen [Pe1, Pe2], there are some nilpotent Lie algebras for which Proposition 3, and more generally Theorem 2, cannot be applied to compute the TP-cohomology of the union $\Omega$ of all maximal dimensional coadjoint orbits. To deal with these cases which are actually the most fascinating, we propose first to examine the example of $\mathfrak{g}=\mathfrak{g}_{4,1}$. The brackets of this filiform Lie algebra are

$$
\left[X_{4}, X_{3}\right]=X_{2}, \quad\left[X_{4}, X_{2}\right]=X_{1} .
$$

Let us identify $\mathfrak{g}^{*}$ with $\mathbb{R}^{4}$ by means of the coordinates system $\left(x_{i}\right)$ of $\mathfrak{g}^{*}$ associated to the basis $\left(X_{i}\right)$. The 2-dimensional orbits in $\mathfrak{g}^{*}$ are of two kinds. There are first the orbits of the points $\mu=\left(\mu_{1}, \mu_{2}, \mu_{3}, \mu_{4}\right)$ with $\mu_{1} \neq 0$, which are parabolic cylinders of the form

$$
O_{\mu}=\left\{\left(\mu_{1}, s, \frac{2 \mu_{1} \mu_{3}-\mu_{2}^{2}+s^{2}}{2 \mu_{1}}, \mu_{1} t\right):(s, t) \in \mathbb{R}^{2}\right\} .
$$

Moreover, for the limiting case $\left(\mu_{1}=0\right)$, there are the orbits of the points $\mu=\left(0, \mu_{2}, \mu_{3}, \mu_{4}\right)$ with $\mu_{2} \neq 0$, which are affine varieties of the form

$$
O_{\mu}=\left\{\left(0, \mu_{2}, s, \mu_{2} t\right):(s, t) \in \mathbb{R}^{2}\right\} .
$$

In this example, the regular Poisson manifold $(\Omega, \Lambda)$ is thus the set

$$
\Omega=\left\{\left(x_{1}, x_{2}, x_{3}, x_{4}\right) \in \mathfrak{g}^{*}: x_{1}^{2}+x_{2}^{2} \neq 0\right\},
$$

endowed with the regular Poisson structure $\Lambda$ coming from the Lie bracket. It is clear that the generic invariants associated to the basis $\left(X_{i}\right)$, namely 


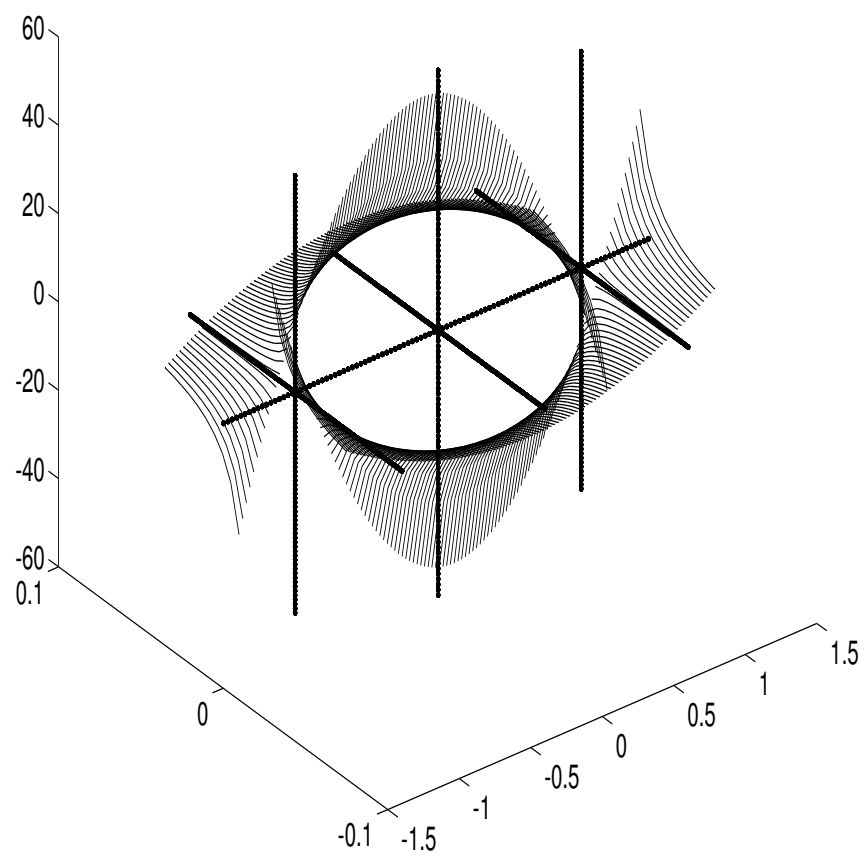

Figure 1. Orbits of the points $(0,1,0,0),(0,-1,0,0)$ and $(\alpha, 1,0,0)$ where $0<|\alpha|<0,1$.

$z_{1}=x_{1}$ and $z_{2}=x_{2}^{2}-2 x_{1} x_{3}$, do not separate the 2-dimensional orbits. In fact, the space of leaves $\Omega / G$ (where $G$ stands for the Lie group corresponding to $\mathfrak{g}$ ) equipped with the quotient topology is not Hausdorff. To see this, consider the orbits $O^{+}$and $O^{-}$of the points $(0,1,0,0)$ and $(0,-1,0,0)$. Denote by $\Pi$ the projection $\Pi: \Omega \rightarrow \Omega / G$. Let $U$ (resp. $V$ ) be any neighborhood of $O^{+}\left(\operatorname{resp} . O^{-}\right)$. Then $\Pi^{-1}(U)$ (resp. $\left.\Pi^{-1}(V)\right)$ is an open subset of $\Omega$ cointaining $(0,1,0,0)$ (resp. $(0,-1,0,0))$. As illustrated by Figure 1, $\Pi^{-1}(U) \cap \Pi^{-1}(V)$ intersects the orbits of the points $(\alpha, 1,0,0)$ for sufficiently small $\alpha$. It follows that $U \cap V=\Pi\left(\Pi^{-1}(U) \cap \Pi^{-1}(V)\right)$ is not empty.

Let us now study the TP-cohomology of $(\Omega, \Lambda)$. As always,

$$
H_{\Lambda, \tan }^{0}(\Omega)=I(\Omega)
$$


To describe $H_{\Lambda, \tan }^{1}(\Omega)$, we shall observe that any tangential vector field $X$ can be written in the form $X=a \partial_{4}+b H_{x_{4}}$ where $a, b$ are in $C^{\infty}(\Omega)$ and that, for such a $X, \sigma(X)=0$ if and only if

$$
H_{x_{4}}(b)+\partial_{4}(a)=x_{2} \partial_{3}(b)+x_{1} \partial_{2}(b)+\partial_{4}(a)=0 .
$$

Let us reduce the study of $H_{\Lambda, \tan }^{1}(\Omega)$ to the resolution of the partial differential equation

$$
H_{x_{4}}(g)=a_{0}
$$

when $a_{0}$ and $g$ depend only on the variables $x_{1}, x_{2}, x_{3}$.

Suppose that $X=a \partial_{4}+b H_{x_{4}}$ satisfies $\sigma(X)=0$, that is (*) holds. Then, there exists $f$ in $C^{\infty}(\Omega)$ such that $X=\sigma f$ if and only if

$$
X\left(d x_{i}\right)=\sigma f\left(d x_{i}\right)=-\left\{f, x_{i}\right\} \quad \forall i,
$$

or equivalently, if and only if

$$
b=-\partial_{4}(f) \quad \text { and } \quad a=H_{x_{4}}(f) .
$$

Thus, the existence of $f$ is equivalent to the existence of $g(x)=g\left(x_{1}, x_{2}, x_{3}\right)$ such that

$$
f(x)=-\int_{0}^{x_{4}} b\left(x_{1}, x_{2}, x_{3}, t\right) d t+g\left(x_{1}, x_{2}, x_{3}\right)
$$

and

$$
H_{x_{4}}(f)(x)=-\int_{0}^{x_{4}} H_{x_{4}} b\left(x_{1}, x_{2}, x_{3}, t\right) d t+H_{x_{4}}(g)(x)=a(x) .
$$

Because of $(*)$, it exactly means that

$$
\begin{aligned}
H_{x_{4}}(f)(x) & =\int_{0}^{x_{4}} \partial_{4}(a)\left(x_{1}, x_{2}, x_{3}, t\right) d t+H_{x_{4}}(g)(x) \\
& =a\left(x_{1}, x_{2}, x_{3}, x_{4}\right)-a\left(x_{1}, x_{2}, x_{3}, 0\right)+H_{x_{4}}(g)(x) \\
& =a\left(x_{1}, x_{2}, x_{3}, x_{4}\right),
\end{aligned}
$$

and thus, as announced, that $H_{x_{4}}(g)(x)=a\left(x_{1}, x_{2}, x_{3}, 0\right)$. We want now to prove:

Lemma 3. Let a be a function in $C^{\infty}(\Omega)$ depending only on the variables $x_{1}, x_{2}, x_{3}$. Assume there exists a function $g$ in $C^{\infty}(\Omega)$, depending also on the variables $x_{1}, x_{2}, x_{3}$, such that

$$
H_{x_{4}}(g)=x_{2} \partial_{3}(g)+x_{1} \partial_{2}(g)=a .
$$

Then,

$$
\lim _{x_{1} \rightarrow 0} \int_{-1}^{1} a\left(x_{1}, s,-\frac{1}{2 x_{1}}+\frac{s^{2}}{2 x_{1}}\right) \frac{d s}{x_{1}}
$$

exists. 
Proof. Using the change of variables on the open set $U=\left\{x \in \Omega: x_{1} \neq 0\right\}$,

$$
u=x_{3}-\frac{x_{2}^{2}}{2 x_{1}}, v=\frac{x_{2}}{x_{1}}, w=x_{1},
$$

we can see that $g$ is necessarily of the form

$$
\begin{aligned}
g\left(x_{1}, x_{2}, x_{3}\right) & =\int_{0}^{\frac{x_{2}}{x_{1}}} a\left(x_{1}, x_{1} t, x_{3}-\frac{x_{2}^{2}}{2 x_{1}}+\frac{t^{2} x_{1}}{2}\right) d t+c\left(x_{1}, x_{3}-\frac{x_{2}^{2}}{2 x_{1}}\right) \\
& =\int_{0}^{x_{2}} a\left(x_{1}, s, x_{3}-\frac{x_{2}^{2}}{2 x_{1}}+\frac{s^{2}}{2 x_{1}}\right) \frac{d s}{x_{1}}+c\left(x_{1}, x_{3}-\frac{x_{2}^{2}}{2 x_{1}}\right) .
\end{aligned}
$$

Thus,

$$
g\left(x_{1}, 1,0\right)=\int_{0}^{1} a\left(x_{1}, s,-\frac{1}{2 x_{1}}+\frac{s^{2}}{2 x_{1}}\right) \frac{d s}{x_{1}}+c\left(x_{1},-\frac{1}{2 x_{1}}\right)
$$

and $g\left(x_{1},-1,0\right)=\int_{0}^{-1} a\left(x_{1}, s,-\frac{1}{2 x_{1}}+\frac{s^{2}}{2 x_{1}}\right) \frac{d s}{x_{1}}+c\left(x_{1},-\frac{1}{2 x_{1}}\right)$.

Therefore, $g\left(x_{1}, 1,0\right)-g\left(x_{1},-1,0\right)=\int_{-1}^{1} a\left(x_{1}, s,-\frac{1}{2 x_{1}}+\frac{s^{2}}{2 x_{1}}\right) \frac{d s}{x_{1}}$. We get the result from the fact that $g$ is continuous at the points $(0,1,0)$ and $(0,-1,0)$ (see also Figure 1).

While the leaves of $\Omega$ are cohomologically trivial, the first TP-cohomology space of $\Omega$ is very large. Indeed, we have:

Proposition 4. Let $\Omega$ be the set of maximal dimensional orbits associated to $\mathfrak{g}=\mathfrak{g}_{4,1}$ and $\Lambda$ its regular Poisson structure. Let $G$ be the connected and simply connected Lie group with Lie algebra $\mathfrak{g}$. Denote by $S(\mathfrak{g})^{G}$ (resp. $\mathcal{A}(\mathfrak{g})^{G}$ ) the ring of $G$-invariant polynomial (resp. analytic) functions over $\mathfrak{g}^{*}$. For each $\alpha>\frac{1}{2}$, denote by $T_{\alpha}$ and $K_{\alpha}$ the vector fields defined by $T_{\alpha}=t_{\alpha} \partial_{4}$ and $K_{\alpha}=k_{\alpha} \partial_{4}$ where

$$
t_{\alpha}=\frac{1}{\left(x_{1}^{2}+x_{2}^{2}\right)^{\alpha}} \quad \text { and } \quad k_{\alpha}=\frac{x_{1} \exp \left(\frac{\alpha}{x_{1}^{2}+x_{2}^{2}}\right)}{\left(x_{1}^{2}+x_{2}^{2}\right)^{2}} .
$$

Then, the following assertions hold.

(i) The classes $\left[T_{\alpha}\right]$ generate an infinite dimensional space over $\mathbb{R}$.

(ii) The classes $\left[K_{\alpha}\right]$ are linearly independent not only over $\mathbb{R}$ but also over $S(\mathfrak{g})^{G}$ or over $\mathcal{A}(\mathfrak{g})^{G}$, i.e., $H_{\Lambda, \tan }^{1}(\Omega)$, as a $S(\mathfrak{g})^{G}$-module or as a $\mathcal{A}(\mathfrak{g})^{G}$-module, is not finitely generated.

Remark 4 (and convention). We use in (ii) the integral domains $S(\mathfrak{g})^{G}$ and $\mathcal{A}(\mathfrak{g})^{G}$, but, from a differential geometry point of view, it would be more interesting to consider the whole ring $I(\Omega)$ of $G$-invariant smooth functions over $\Omega$. In fact, due to the complexity of the non-integral domain $I(\Omega)$, 
we do not know the $I(\Omega)$-module structure of $H_{\Lambda, \tan }^{1}(\Omega)$. Nevertheless we conjecture that it is still not finitely generated.

In the sequel, when we say for some Lie algebra $\mathfrak{g}$ that a TP-cohomology (or a P-cohomology) space of $\Omega$ is infinite dimensional, we will mean both infinite dimensional as a vector space and not finitely generated as a module over $S(\mathfrak{g})^{G}$ or $\mathcal{A}(\mathfrak{g})^{G}$.

Proof of Proposition 4. For all $0<x_{1}<1$, we have

$$
\int_{0}^{1} \frac{d s}{\left(x_{1}^{2}+s^{2}\right)^{\alpha} x_{1}} \geq \int_{0}^{x_{1}} \frac{d s}{\left(x_{1}^{2}+s^{2}\right)^{\alpha} x_{1}} \geq \frac{1}{2^{\alpha} x_{1}^{2 \alpha}}
$$

and

$$
\begin{aligned}
\int_{0}^{1} \frac{d s}{\left(x_{1}^{2}+s^{2}\right)^{\alpha} x_{1}} & \leq \int_{0}^{x_{1}} \frac{d s}{\left(x_{1}^{2}+s^{2}\right)^{\alpha} x_{1}}+\int_{x_{1}}^{1} \frac{d s}{s^{2 \alpha} x_{1}} \\
& \leq c_{\alpha} \frac{1}{x_{1}^{2 \alpha}} \quad\left(\forall \alpha>\frac{1}{2}\right)
\end{aligned}
$$

where $c_{\alpha}=\frac{2 \alpha}{2 \alpha-1}$. Now, assume that for some $p, \sum_{i=1}^{p} \lambda_{\alpha_{i}}\left[T_{\alpha_{i}}\right]=0$, the $\lambda_{\alpha_{i}}$ being in $\mathbb{R}$ and $\alpha_{1}<\ldots<\alpha_{p}$. Using Lemma 3, we directly see that

$$
E=\int_{0}^{1} \sum_{i=1}^{p} \lambda_{\alpha_{i}} \frac{d s}{\left(x_{1}^{2}+s^{2}\right)^{\alpha_{i}} x_{1}}
$$

must have a limit when $x_{1}$ tends to zero. But, when $0<x_{1}<1$,

$$
\begin{aligned}
|E| \geq\left|\int_{0}^{1} \frac{\lambda_{\alpha_{p}} d s}{x_{1}\left(x_{1}^{2}+s^{2}\right)^{\alpha_{p}}}\right|-\left|\int_{0}^{1} \frac{\lambda_{\alpha_{p-1}} d s}{x_{1}\left(x_{1}^{2}+s^{2}\right)^{\alpha_{p-1}}}\right| \\
\ldots-\left|\int_{0}^{1} \frac{\lambda_{\alpha_{1}} d s}{x_{1}\left(x_{1}^{2}+s^{2}\right)^{\alpha_{1}}}\right| \\
\geq \frac{\left|\lambda_{\alpha_{p}}\right|}{2^{\alpha_{p}} x_{1}^{2 \alpha_{p}}}-\frac{\left|\lambda_{\alpha_{p-1}}\right| c_{\alpha_{p-1}}}{x_{1}^{2 \alpha_{p-1}}}-\cdots-\frac{\left|\lambda_{\alpha_{1}}\right| c_{\alpha_{1}}}{x_{1}^{2 \alpha_{1}}} .
\end{aligned}
$$

Thus, $\lambda_{\alpha_{p}}$ must be 0 and a step-by-step application of the same argument shows that $\lambda_{\alpha_{i}}=0$ for all $i$. It implies that the classes $\left[T_{\alpha}\right]$ generate an infinite dimensional vector space over $\mathbb{R}$. That ends the proof of (i).

To prove (ii), it is enough to see that

$$
\begin{aligned}
\int_{0}^{1} \frac{2 \exp \left(\frac{\alpha}{x_{1}^{2}+s^{2}}\right)}{\left(x_{1}^{2}+s^{2}\right)^{2}} d s & \geq \int_{0}^{1} \frac{2 s \exp \left(\frac{\alpha}{x_{1}^{2}+s^{2}}\right)}{\left(x_{1}^{2}+s^{2}\right)^{2}} d s \\
& \geq \frac{1}{\alpha}\left(\exp \left(\frac{\alpha}{x_{1}^{2}}\right)-\exp \left(\frac{\alpha}{x_{1}^{2}+1}\right)\right),
\end{aligned}
$$


and that

$$
\int_{0}^{1} \frac{2 \exp \left(\frac{\alpha}{x_{1}^{2}+s^{2}}\right)}{\left(x_{1}^{2}+s^{2}\right)^{2}} d s \leq \frac{2 \exp \left(\frac{\alpha}{x_{1}^{2}}\right)}{x_{1}^{4}} .
$$

Thus we can use the same argument as in (i).

To finish the discussion about this example, we shall prove the vanishing of $H_{\Lambda, \tan }^{2}(\Omega)$. Let $A$ be a tangential 2-tensor field. Necessarily,

$$
A=\varphi \Lambda=\varphi\left(x_{2} \partial_{4} \wedge \partial_{3}+x_{1} \partial_{4} \wedge \partial_{2}\right)
$$

for some function $\varphi$ in $C^{\infty}(\Omega)$ and $\sigma(A)=0$. We have thus to find a tangential vector field $B$ such that $A=\sigma(B)$, or equivalently, to find $a, b$ in $C^{\infty}(\Omega)$ such that $H_{x_{4}}(b)+\partial_{4}(a)=\varphi$. We immediately check that

$$
a=\int_{0}^{x_{4}} \varphi\left(x_{1}, x_{2}, x_{3}, t\right) d t \quad \text { and } \quad b=0
$$

are convenient. Therefore, $H_{\Lambda, \tan }^{2}(\Omega)=\{0\}$.

This fact can also be deduced from Proposition 1 (Section 2), which identifies the TP-cogomology with a $\check{C}$ ech cohomology. Indeed, it is easy to see that $\Omega$ admits a good covering $\mathcal{U}=\left(U_{i}\right)$ without any nontrivial intersections of three open sets $U_{i}$.

Now, since the rank of $\Omega$ is 2 , we have $H_{\Lambda, \tan }^{k}(\Omega)=\{0\}$ for all $k>2$.

Remark 5. An immediate consequence of the above analysis concerning the nilpotent case is that the tangential star products on $\cup_{B} V_{B}$ (and on any regular Poisson manifold $\widetilde{\Omega}$ satisfying the conditions of Proposition 3) are all equivalent because of the vanishing of the second TP-cohomology space (Propositions 2 and 3). The same is true for the union $\Omega$ of all maximal dimensional coadjoint orbits in $\mathfrak{g}_{4,1}^{*}$.

The computation of the TP-cohomology spaces for $\mathfrak{g}_{5,4}, \mathfrak{g}_{6,18}$ or for all the filiform Lie algebras, studied for instance in [Pe1, Pe2, CG, GK, BLM], leads to the same results as in the $\mathfrak{g}_{4,1}$-case. Due to these examples, we believe that the TP-cohomology spaces of a regular Poisson manifold $M$ are huge and rather complicated to compute whenever the quotient space of $M$ by the foliation is not Hausdorff. To confirm this observation, we shall now study more varied examples.

\section{Further examples.}

Let $(M, \Lambda)$ be a 3 -dimensional regular Poisson manifold. If we exclude the trivial case where $\Lambda=0$, we can suppose $M$ to be of rank 2. To describe the P-cohomology of $M$, we are going to use Vaisman's notations and computations ([Va2, p. 69]). Of course,

$$
H_{\Lambda}^{0}(M)=H_{\Lambda, \tan }^{0}(M)=I(M) .
$$


Now, we have by definition

$$
H_{\Lambda}^{1}(M)=\frac{\left\{Q \in \mathcal{V}^{1}(M): \sigma(Q)=0\right\}}{\left\{\sigma f: f \in C^{\infty}(M)\right\}} .
$$

Let us then choose a transversal distribution $\nu \mathcal{F}$. Let us also use the decompositions $\underset{q}{\oplus} \mathcal{V}_{0, q}(M), \underset{q}{\oplus} \Omega_{0, q}(M), \sigma=\sigma^{\prime}+\sigma^{\prime \prime}$ and $d=d^{\prime}+d^{\prime \prime}+d_{2,-1}$. Each element $Q$ of $\mathcal{V}^{1}(M)$ can thus be written in the form

$$
Q=Q_{0,1}+Q_{1,0}
$$

where $Q_{0,1}$ and $Q_{1,0}$ are of type $(0,1)$ and $(1,0)$ respectively. Furthermore, $\sigma(Q)=0$ if and only if $\sigma\left(Q_{0,1}+Q_{1,0}\right)=0$, or else, if and only if

$$
\sigma^{\prime}\left(Q_{1,0}\right)+\sigma^{\prime \prime}\left(Q_{1,0}\right)+\sigma^{\prime \prime}\left(Q_{0,1}\right)=0,
$$

that is, if and only if

$$
\sigma^{\prime \prime}\left(Q_{1,0}\right)=0 \quad \text { and } \quad \sigma^{\prime}\left(Q_{1,0}\right)+\sigma^{\prime \prime}\left(Q_{0,1}\right)=0 .
$$

Consider now the linear mapping $p$ defined by

$$
\begin{aligned}
p: H_{\Lambda}^{1}(M) & \longrightarrow \widetilde{\mathcal{V}}_{1,0}(M) \\
{[A] } & \longmapsto A_{1,0}
\end{aligned}
$$

where

$$
\widetilde{\mathcal{V}}_{1,0}(M)=\left\{A \in \mathcal{V}_{1,0}(M): \sigma^{\prime \prime}(A)=0\right\}
$$

It follows that

$$
H_{\Lambda}^{1}(M) \cong \operatorname{Ker}(p) \oplus \operatorname{Im}(p)
$$

Now

$$
\begin{aligned}
\operatorname{Ker}(p) & =\left\{[A] \in H_{\Lambda}^{1}(M): A_{1,0}=0\right\} \\
& =\frac{\left\{A \in \mathcal{V}_{0,1}(M): \sigma(A)=0\right\}}{\left\{\sigma f: f \in C^{\infty}(M)\right\}} \\
& =\frac{\left\{A \in \mathcal{V}_{0,1}(M): \sigma^{\prime \prime}(A)=0\right\}}{\left\{\sigma^{\prime \prime} f: f \in C^{\infty}(M)\right\}} \\
& =H_{\Lambda, \tan }^{1}(M) .
\end{aligned}
$$

Moreover,

$$
\begin{aligned}
\operatorname{Im}(p)=\left\{A \in \mathcal{V}_{1,0}(M): \sigma^{\prime \prime}(A)=0\right. & \text { and } \\
& \left.\exists B \in \mathcal{V}_{0,1}(M) / \sigma(A)+\sigma^{\prime \prime}(B)=0\right\} .
\end{aligned}
$$


Let us compute the second order space:

$$
\begin{aligned}
& H_{\Lambda}^{2}(M)=\frac{\left\{Q \in \mathcal{V}^{2}(M): \sigma(Q)=0\right\}}{\left\{\sigma(V): V \in \mathcal{V}^{1}(M)\right\}} \\
& =\frac{\left\{Q=Q_{0,2}+Q_{1,1} \in \mathcal{V}^{2}(M): \sigma^{\prime \prime}\left(Q_{1,1}\right)=0\right\}}{\left\{\sigma(V): V=V_{0,1}+V_{1,0} \in \mathcal{V}^{1}(M)\right\}} \\
& =\frac{\left\{Q_{0,2} \in \mathcal{V}_{0,2}(M)\right\}}{\left\{\sigma^{\prime \prime}\left(V_{0,1}\right)+\sigma^{\prime}\left(V_{1,0}\right)\right\}} \oplus \frac{\left\{Q_{1,1} \in \mathcal{V}_{1,1}(M): \sigma^{\prime \prime}\left(Q_{1,1}\right)=0\right\}}{\left\{\sigma^{\prime \prime}\left(V_{1,0}\right)\right\}} \\
& =\frac{\frac{\left\{Q_{0,2}\right\}}{\left\{\sigma^{\prime \prime}\left(V_{0,1}\right)\right\}}}{\sigma\left(\mathcal{V}_{1,0}(M)\right)} \oplus \frac{\left\{Q_{1,1}: \sigma^{\prime \prime}\left(Q_{1,1}\right)=0\right\}}{\left\{\sigma^{\prime \prime}\left(V_{1,0}\right)\right\}} \\
& =\frac{H_{\Lambda, \tan }^{2}(M)}{\sigma\left(\mathcal{V}_{1,0}(M)\right)} \oplus \frac{\operatorname{Ker}\left(\sigma_{\mid \mathcal{V}_{1,1}(M)}^{\prime \prime}\right)}{\sigma^{\prime \prime}\left(\mathcal{V}_{1,0}(M)\right)}
\end{aligned}
$$

In the same way, we get the third order space:

$$
H_{\Lambda}^{3}(M)=\frac{\left\{Q_{1,2}\right\}}{\left\{\sigma\left(V_{0,2}+V_{1,1}\right)\right\}}=\frac{\left\{Q_{1,2}\right\}}{\left\{\sigma^{\prime \prime}\left(V_{1,1}\right)\right\}}=\frac{\mathcal{V}_{1,2}(M)}{\sigma^{\prime \prime}\left(\mathcal{V}_{1,1}(M)\right)} .
$$

As seen in Section 2, for all regular Poisson manifold $(M, \Lambda),\left(\underset{q}{\oplus} \Omega_{0, q}(M)\right.$, $\left.d^{\prime \prime}\right)$ and $\left(\underset{q}{\oplus} \mathcal{V}_{0, q}(M), \sigma^{\prime \prime}\right)$ are isomorphic as complexes of $I(M)$-modules. In fact, it is always possible to define an isomorphism between $\Omega_{p, q}(M)$ and $\mathcal{V}_{p, q}(M)$ for all $p$ and $q$. For this, one can consider, as Vaisman did in [Va2], an Euclidean metric on $\nu^{*} \mathcal{F}$. That leads to an isomorphism between $\nu^{*} \mathcal{F} \oplus T^{*} \mathcal{F}$ and $\nu \mathcal{F} \oplus T \mathcal{F}$, which can naturally be extended to the required isomorphism between $\Omega_{p, q}(M)$ and $\mathcal{V}_{p, q}(M)$. In general, however, this isomorphism is not an isomorphism of complexes. Via the next proposition, we give a simple situation where $\left(\underset{q}{\oplus} \Omega_{p, q}(M), d^{\prime \prime}\right)$ and $\left(\underset{q}{\oplus} \mathcal{V}_{p, q}(M), \sigma^{\prime \prime}\right)$ are effectively isomorphic complexes for all $p$.

Proposition 5. Let $(M, \Lambda)$ be a regular Poisson manifold. Denote by $\mathcal{F}$ the symplectic foliation of $M$. Choose a transversal distribution $\nu \mathcal{F}$ and assume that $\Omega_{1,0}(M)$ and $\mathcal{V}_{1,0}(M)$ are isomorphic free $I(M)$-modules with bases $\left(\beta_{i}\right)$ and $\left(X_{i}\right)$ respectively $\left(\beta_{i}\right.$ and $X_{i}$ being globally defined). Suppose also that $d^{\prime \prime} \beta_{i}=0$ and $\sigma^{\prime \prime}\left(X_{i}\right)=0$. Then, for all $p,\left(\underset{q}{\oplus} \Omega_{p, q}(M), d^{\prime \prime}\right)$ and $\left(\oplus_{q} \mathcal{V}_{p, q}(M), \sigma^{\prime \prime}\right)$ are isomorphic complexes of $I(M)$-modules. In particular, $H^{q}\left(M, \Phi^{p}(\mathcal{F})\right)$ and $H^{q}\left(\underset{k}{\oplus} \mathcal{V}_{p, k}(M), \sigma^{\prime \prime}\right)$ are isomorphic as $I(M)$-modules. 
Proof. Recall that \# : $T^{*} M \rightarrow T M$ can be extended to an $I(M)$-modules isomorphism $\widetilde{\#}: \Omega_{0, q}(M) \rightarrow \mathcal{V}_{0, q}(M)$ satisfying $\sigma^{\prime \prime} \circ \widetilde{\#}=-\widetilde{\#} \circ d^{\prime \prime}$. Consider now the mapping $\hat{\#}: \Omega_{p, q}(M) \rightarrow \mathcal{V}_{p, q}(M)$ defined by

$$
\hat{\#}\left(\sum_{i_{1}, \ldots, i_{p}} \alpha_{i_{1}, \ldots, i_{p}} \wedge \beta_{i_{1}} \wedge \ldots \wedge \beta_{i_{p}}\right)=\sum_{i_{1}, \ldots, i_{p}}\left(\widetilde{\#}\left(\alpha_{i_{1}, \ldots, i_{p}}\right) \wedge X_{i_{1}} \wedge \ldots \wedge X_{i_{p}}\right)
$$

where the $\alpha_{i_{1}, \ldots, i_{p}}$ are in $\Omega_{0, q}(M)$. It is not difficult to check that $\hat{\#}$ is an $I(M)$-modules isomorphism and that $\sigma^{\prime \prime} \circ \hat{\#}=-\hat{\#} \circ d^{\prime \prime}$. This ends the proof.

The following definitions are quite standard, we recall them for completeness. Let $V \rightarrow B$ be a vector bundle whose fibers are $q$-dimensional. We shall say that $V \rightarrow B$ (or simply $V$ ) is orientable if the bundle $\Lambda^{q} V \rightarrow B$ admits a global nonsingular (i.e., nowhere vanishing) section. If $V \rightarrow B$ is orientable, so is its dual $V^{*} \rightarrow B$. Recall also that a manifold $M$ is said to be orientable if $T M$ (or $T^{*} M$ ) is orientable.

Moreover, we shall say that a foliation $\mathcal{F}$ on $M$ is (co)orientable if its normal bundle $N \mathcal{F}$ is orientable. In the important case of a 1-codimensional foliation $\mathcal{F}$, this foliation $\mathcal{F}$ is orientable if and only if there exists a nonsingular 1-form $\beta$ vanishing exactly on vectors tangent to the leaves of $\mathcal{F}$. In this case, we say that $\beta$ defines the foliation.

Note that neither the leaves nor the total manifold $M$ of an orientable foliation need to be orientable. However, if $(M, \Lambda)$ is a regular Poisson manifold (of rank $2 n$ ) and $\mathcal{F}$ is the symplectic foliation of $M$, then the situation is somewhat simpler. Since the tensor $\Lambda^{n}$ defines a global nonsingular section of $\Lambda^{2 n} T \mathcal{F}$, the tangent bundle $T \mathcal{F}$ of $\mathcal{F}$ is orientable. In other words, the symplectic foliation of a regular Poisson manifold $M$ is orientable if and only if $M$ is orientable.

We turn back now to the case where $(M, \Lambda)$ is 3 -dimensional and give a result which will be useful later.

Proposition 6. Let $(M, \Lambda)$ be a regular Poisson manifold of dimension 3 and rank 2. Denote by $\mathcal{F}$ the symplectic foliation of $M$. Suppose that $M$ is orientable and that one can choose the 1 -form $\beta$ defining $\mathcal{F}$ such that $d \beta=0$. Then:

(i) The second and third P-cohomology spaces of $M$ are

$$
\begin{aligned}
H_{\Lambda}^{2}(M) & \simeq \frac{H_{\Lambda, \tan }^{2}(M)}{\sigma\left(\mathcal{V}_{1,0}(M)\right)} \oplus H^{1}\left(M, \Phi^{1}(\mathcal{F})\right) \\
H_{\Lambda}^{3}(M) & \simeq H^{2}\left(M, \Phi^{1}(\mathcal{F})\right) .
\end{aligned}
$$

(ii) For all $q, H_{\Lambda, \tan }^{q}(M)$ and $H^{q}\left(M, \Phi^{1}(\mathcal{F})\right)$ are isomorphic as $I(M)$ modules. 
Proof. Let us identify the normal bundle $N \mathcal{F}$ of $\mathcal{F}$ with a sub-bundle $\nu \mathcal{F}$ of $T M$. Since $M$ (or $\mathcal{F}$ ) is orientable, there exists a nonsingular vector field $X$ (globally defined) such that, for each $x, \nu_{x} \mathcal{F}$ (resp. $\nu_{x}^{*} \mathcal{F}$ ) is spanned by $X_{x}$ (resp. $\beta_{x}$ ). By construction, $\sigma^{\prime \prime} X=0$ and $d \beta=d^{\prime \prime} \beta=0$. Moreover, $\Omega_{1,0}(M)$ and $\mathcal{V}_{1,0}(M)$ are isomorphic free and with basis $(\beta)$ and $(X)$ respectively. The point (i) is thus a direct corollary of Proposition 5.

To prove (ii), denote by $\Phi: H^{q}\left(M, \Phi^{0}(\mathcal{F})\right) \rightarrow H^{q}\left(M, \Phi^{1}(\mathcal{F})\right)$ the mapping defined by $\Phi([\alpha])=[\alpha \wedge \beta]$ for all $\alpha$ in $\Omega_{0, q}(M)$ such that $d^{\prime \prime} \alpha=0$. Clearly, $\Phi$ is both well-defined and bijective. Therefore, $H^{q}\left(M, \Phi^{0}(\mathcal{F})\right.$ ) (which is isomorphic to $H_{\Lambda, \tan }^{q}(M)$ by Theorem 1$)$ coincides with $H^{q}\left(M, \Phi^{1}(\mathcal{F})\right)$. This ends the proof.

As we already said, each Lie algebra provides a natural regular Poisson manifold: The union $\Omega$ of all maximal dimensional coadjoint orbits. Let us now study the TP-cohomology and the P-cohomology of $\Omega$ for any 3 dimensional Lie algebra.

First recall that every nonabelian 3-dimensional Lie algebra is isomorphic to exactly one in the following list (see $[\mathbf{B r}]$ for instance):

- a nilpotent Lie algebra, namely the 3-dimensional Heisenberg algebra, given by: $\left[X_{1}, X_{2}\right]=X_{3}$;

- a solvable non-exponential Lie algebra, namely $\mathfrak{e}(2)$, defined by: $\left[X_{1}, X_{2}\right]=-X_{3},\left[X_{1}, X_{3}\right]=X_{2}$;

- several (solvable) exponential Lie algebras:

* the algebra spanned by $X_{1}, X_{2}, X_{3}$ with $\left[X_{1}, X_{2}\right]=X_{2}$;

* the "book algebra":

$\left[X_{1}, X_{2}\right]=X_{2},\left[X_{1}, X_{3}\right]=X_{3}$;

* "Grélaud's Lie algebras":

$\left[X_{1}, X_{2}\right]=X_{2}-\sigma X_{3},\left[X_{1}, X_{3}\right]=X_{3}+\sigma X_{2}$ where $\sigma>0$;

$*\left[X_{1}, X_{2}\right]=X_{2},\left[X_{1}, X_{3}\right]=\frac{1}{\tau} X_{3}$ where $\tau>1$;

$*\left[X_{1}, X_{2}\right]=X_{2}+X_{3},\left[X_{1}, X_{3}\right]=X_{3}$;

$*\left[X_{1}, X_{2}\right]=X_{2},\left[X_{1}, X_{3}\right]=-X_{3}$;

$*\left[X_{1}, X_{2}\right]=X_{2},\left[X_{1}, X_{3}\right]=\frac{1}{\tau} X_{3}$ where $\tau<-1$;

- two simple Lie algebras:

$\mathfrak{s u}(2)$ and $\mathfrak{s l}(2)$.

Among them, there are some easy examples. Let us pass them in review.

4.1. The 3-dimensional Heisenberg algebra. This nilpotent Lie algebra is defined by the bracket $\left[X_{1}, X_{2}\right]=X_{3}$.

For this example, the nontrivial orbits are planes and the regular Poisson manifold $(\Omega, \Lambda)$ is the set

$$
\Omega=\left\{\left(x_{1}, x_{2}, x_{3}\right): x_{3} \neq 0\right\},
$$


endowed with its regular Poisson structure $\Lambda$. We identify $\Omega$ with $\mathbb{R}^{2} \times \mathbb{R}^{*}$ by means of the Weinstein chart [SG, Wei] mapping an element $x=\left(x_{1}, x_{2}, x_{3}\right)$ of $\Omega$ to $\left(p=x_{1}, q=\frac{x_{2}}{x_{3}}, z=x_{3}\right)$.

Using Theorem 3 (or Theorem 2), we see that the TP-cohomology of $\Omega$ is trivial in degree superior to zero.

Furthermore, $\Lambda$ is transversally constant with respect to the transversal distribution $\nu \mathcal{F}=T \mathbb{R}^{*}$. Using Theorem 4 or by direct computation, we get the P-cohomology of $\Omega$ :

$$
\begin{aligned}
& H_{\Lambda}^{0}(\Omega)=H_{\Lambda, \tan }^{0}(\Omega)=I(\Omega)=C^{\infty}\left(\mathbb{R}^{*}\right) \\
& H_{\Lambda}^{1}(\Omega) \simeq\{u d z: u \in I(\Omega)\} \simeq I(\Omega) \\
& H_{\Lambda}^{k}(\Omega)=\{0\} \quad \forall k>1 .
\end{aligned}
$$

4.2. The Lie-algebra $\mathfrak{e}(2)$ of the Euclidean 2-dimensional group. The brackets are:

$$
\left[X_{1}, X_{2}\right]=-X_{3}, \quad\left[X_{1}, X_{3}\right]=X_{2} .
$$

The nontrivial orbits are cylinders $C_{r}$ with radius $r>0$, and the regular Poisson manifold $(\Omega, \Lambda)$ associated to this Lie algebra is the set

$$
\Omega=\left\{\left(x_{1}, x_{2}, x_{3}\right) \in \mathfrak{e}(2)^{*}: x_{2}^{2}+x_{3}^{2} \neq 0\right\},
$$

endowed with its regular Poisson structure $\Lambda$. We identify $\Omega$ with $\mathbb{R} \times \mathbb{T} \times \mathbb{R}_{+}^{*}$ by means of the Weinstein chart mapping an element $x=\left(x_{1}, x_{2}, x_{3}\right)$ of $\Omega$ to the point $(p, q, r)$ defined by

$$
p=x_{1}, \quad e^{\imath q}=\frac{x_{2}+\imath x_{3}}{\sqrt{x_{2}^{2}+x_{3}^{2}}}, \quad r=\sqrt{x_{2}^{2}+x_{3}^{2}} .
$$

Using Theorem 3, we obtain the TP-cohomology of $\Omega$ :

$$
\begin{aligned}
H_{\Lambda, \tan }^{0}(\Omega) & =I(\Omega)=C^{\infty}\left(\mathbb{R}_{+}^{*}\right) \\
H_{\Lambda, \tan }^{1}(\Omega) & \simeq H_{D R}^{1}(\mathbb{R} \times \mathbb{T}) \otimes C^{\infty}\left(\mathbb{R}_{+}^{*}\right) \\
& \simeq\{[\rho(r) d q]: \rho(r) \in I(\Omega)\} \simeq I(\Omega) \\
H_{\Lambda, \tan }^{k}(\Omega) & =\{0\} \quad \forall k>1 .
\end{aligned}
$$

Now, $\Lambda$ is transversally constant with respect to the transversal distribution $\nu \mathcal{F}=T \mathbb{R}_{+}^{*}$. By Theorem 4, the $\mathrm{P}$-cohomology of $\Omega$ is:

$$
\begin{aligned}
H_{\Lambda}^{0}(\Omega) & =I(\Omega) \\
H_{\Lambda}^{1}(\Omega) & \simeq H_{\Lambda, \tan }^{1}(\Omega) \oplus\{u d r: u \in I(\Omega)\} \\
& \simeq I(\Omega) \oplus I(\Omega) \\
H_{\Lambda}^{2}(\Omega) & \simeq H_{D R}^{1}(\mathbb{R} \times \mathbb{T}) \otimes \Omega^{1}\left(\mathbb{R}_{+}^{*}\right) \\
& \simeq\{[\rho(r) d q \wedge d r]: \rho(r) \in I(\Omega)\} \simeq I(\Omega) \\
H_{\Lambda}^{k}(\Omega) & =\{0\} \quad \forall k>2 .
\end{aligned}
$$


4.3. The Lie algebra $\mathfrak{a}$ of the affine group. The only non-vanishing bracket is $\left[X_{1}, X_{2}\right]=X_{2}$.

The nontrivial orbits are half planes $\left(x_{3}\right.$ and $\operatorname{sign}\left(x_{2}\right)$ fixed) and the regular Poisson manifold $(\Omega, \Lambda)$ associated to this Lie algebra is the set

$$
\Omega=\left\{\left(x_{1}, x_{2}, x_{3}\right) \in \mathfrak{a}^{*}: x_{2} \neq 0\right\},
$$

endowed with its regular Poisson structure $\Lambda$. We identify $\Omega$ with $\mathbb{R}^{*} \times \mathbb{R} \times \mathbb{R}$ by means of the Weinstein chart mapping an element $x=\left(x_{1}, x_{2}, x_{3}\right)$ of $\Omega$ to the point $(p, q, z)$ defined by

$$
p=x_{2}, \quad q=\frac{-x_{1}}{x_{2}}, \quad z=x_{3} .
$$

By Theorem 3 (or Theorem 2), the TP-cohomology of $\Omega$ is trivial in degree superior to zero.

Again, $\Lambda$ is transversally constant with respect to the transversal distribution $\nu \mathcal{F}=T \mathbb{R}$. By Theorem 4, the P-cohomology of $\Omega$ is:

$$
\begin{aligned}
H_{\Lambda}^{0}(\Omega) & =H_{\Lambda, \tan }^{0}(\Omega)=I(\Omega) \\
& \simeq C^{\infty}((\{1\} \times \mathbb{R}) \cup(\{-1\} \times \mathbb{R})) \\
& \simeq C^{\infty}(\mathbb{R}) \oplus C^{\infty}(\mathbb{R}) \\
H_{\Lambda}^{1}(\Omega) & \simeq\{u d z: u \in I(\Omega)\} \simeq I(\Omega) \\
H_{\Lambda}^{k}(\Omega) & =\{0\} \quad \forall k>1 .
\end{aligned}
$$

4.4. The book algebra. This Lie algebra is given by the following brackets:

$$
\left[X_{1}, X_{2}\right]=X_{2}, \quad\left[X_{1}, X_{3}\right]=X_{3} .
$$

The 2-dimensional orbits are characterized by an invariant $\theta$ and are of the form

$$
O_{\theta}=\left\{\left(s, e^{t} \cos (\theta), e^{t} \sin (\theta)\right):(s, t) \in \mathbb{R}^{2}\right\} .
$$

The corresponding regular Poisson manifold $(\Omega, \Lambda)$ is thus the set

$$
\Omega=\left\{\left(x_{1}, x_{2}, x_{3}\right): x_{2}^{2}+x_{3}^{2} \neq 0\right\},
$$

with its regular Poisson structure $\Lambda$. We identify $\Omega$ with $\mathbb{R} \times \mathbb{R} \times \mathbb{T}$ by means of the Weinstein chart mapping an element $x=\left(x_{1}, x_{2}, x_{3}\right)$ of $\Omega$ to the point $(p, q, \theta)$ defined by

$$
p=x_{1}, \quad q=\frac{1}{2} \ln \left(x_{2}^{2}+x_{3}^{2}\right), \quad e^{\imath \theta}=\frac{x_{2}+\imath x_{3}}{\sqrt{x_{2}^{2}+x_{3}^{2}}} .
$$

By Theorem 3 (or Theorem 2), the TP-cohomology of $\Omega$ is trivial in degree superior to zero. 
Moreover, $\Lambda$ is transversally constant with respect to $\nu \mathcal{F}=T \mathbb{T}$. By Theorem 4, the P-cohomology of $\Omega$ is:

$$
\begin{aligned}
& H_{\Lambda}^{0}(\Omega)=H_{\Lambda, \tan }^{0}(\Omega)=I(\Omega)=C^{\infty}(\mathbb{T}) \\
& H_{\Lambda}^{1}(\Omega) \simeq\{u d \theta: u \in I(\Omega)\} \simeq I(\Omega) \\
& H_{\Lambda}^{k}(\Omega)=\{0\} \quad \forall k>1 .
\end{aligned}
$$

4.5. Similar to the book algebra: Grélaud's Lie algebras. The brackets of these Lie algebras, studied by Grélaud in [Gré], are

$$
\left[X_{1}, X_{2}\right]=X_{2}-\sigma X_{3}, \quad\left[X_{1}, X_{3}\right]=X_{3}+\sigma X_{2} \quad \sigma>0 .
$$

The 2-dimensional orbits are a "spiral" version of those of the book algebra, they are of the form

$$
O_{\theta}=\left\{\left(s, e^{t} \cos (\theta+\sigma t), e^{t} \sin (\theta+\sigma t)\right):(s, t) \in \mathbb{R}^{2}\right\}
$$

where $\theta$ is defined by

$$
e^{\imath \theta}=\frac{x_{2}+\imath x_{3}}{\sqrt{x_{2}^{2}+x_{3}^{2}}} e^{-\frac{\imath \sigma}{2} \ln \left(x_{2}^{2}+x_{3}^{2}\right)} .
$$

The regular Poisson manifold $(\Omega, \Lambda)$ is thus the set

$$
\Omega=\left\{\left(x_{1}, x_{2}, x_{3}\right): x_{2}^{2}+x_{3}^{2} \neq 0\right\},
$$

with its regular Poisson structure $\Lambda$.

The situation here is identical to that of the book algebra. First, $\Omega$ can be identified with $\mathbb{R} \times \mathbb{R} \times \mathbb{T}$ and the TP-cohomology of $\Omega$ is trivial in degree superior to zero. Moreover, $\Lambda$ is transversally constant with respect to $\nu \mathcal{F}=T \mathbb{T}$ so that the $\mathrm{P}$-cohomology of $\Omega$ is:

$$
\begin{aligned}
& H_{\Lambda}^{0}(\Omega)=H_{\Lambda, \tan }^{0}(\Omega)=I(\Omega)=C^{\infty}(\mathbb{T}) \\
& H_{\Lambda}^{1}(\Omega)=\{u d \theta: u \in I(\Omega)\} \simeq I(\Omega) \\
& H_{\Lambda}^{k}(\Omega)=\{0\} \quad \forall k>1 .
\end{aligned}
$$

4.6. Other examples very close to the book algebra. Consider the family of Lie algebras defined by the brackets:

$$
\left[X_{1}, X_{2}\right]=X_{2}, \quad\left[X_{1}, X_{3}\right]=\frac{X_{3}}{\tau} \quad \tau>1 .
$$

The 2-dimensional orbits can be parameterized with obvious notations by

$$
O_{\mu}=\left\{\left(s, e^{-t} \mu_{2}, e^{-\frac{t}{\tau}} \mu_{3}\right):(s, t) \in \mathbb{R}^{2}\right\},
$$

they are all cohomologically trivial and the regular Poisson manifold $(\Omega, \Lambda)$ is the set

$$
\Omega=\left\{\left(x_{1}, x_{2}, x_{3}\right): x_{2}^{2}+x_{3}^{2} \neq 0\right\}
$$

with its regular Poisson structure $\Lambda$. 
Let us now prove that the symplectic foliation $\mathcal{F}$ of $\Omega$ is given by a submersion $\Pi$ from $\Omega$ to the circle $\mathbb{S}^{1}$. Let

$$
F: \mathbb{R} \times \mathbb{R}^{2} \backslash\{(0,0)\} \rightarrow \mathbb{R}
$$

be the mapping defined by

$$
F\left(t, x_{2}, x_{3}\right)=e^{-2 t} x_{2}^{2}+e^{-\frac{2 t}{\tau}} x_{3}^{2}-1 .
$$

Using the standard Implicit Function Theorem, we see there is a unique smooth function $\varphi: \mathbb{R}^{2} \backslash\{(0,0)\} \rightarrow \mathbb{R}$ of the variables $x_{2}, x_{3}$ such that

$$
F\left(\varphi\left(x_{2}, x_{3}\right), x_{2}, x_{3}\right)=0 \quad \forall\left(x_{2}, x_{3}\right) \in \mathbb{R}^{2} \backslash\{(0,0)\} .
$$

Moreover, the partial derivatives of $\varphi$ are

$$
\begin{aligned}
\frac{\partial \varphi}{\partial x_{2}} & =\frac{e^{-2 \varphi} x_{2}}{e^{-2 \varphi} x_{2}^{2}+e^{\frac{-2 \varphi}{\tau}} \frac{x_{3}^{2}}{\tau}} \\
\frac{\partial \varphi}{\partial x_{3}} & =\frac{e^{\frac{-2 \varphi}{\tau}} x_{3}}{e^{-2 \varphi} x_{2}^{2}+e^{\frac{-2 \varphi}{\tau}} \frac{x_{3}^{2}}{\tau}}
\end{aligned}
$$

It is easy to check that the map $\Pi: \Omega \rightarrow \mathbb{S}^{1}$ defined by

$$
\left(x_{1}, x_{2}, x_{3}\right) \longmapsto \Pi\left(x_{1}, x_{2}, x_{3}\right)=\left(e^{-\varphi\left(x_{2}, x_{3}\right)} x_{2}, e^{\frac{-\varphi\left(x_{2}, x_{3}\right)}{\tau}} x_{3}\right)
$$

is a submersion. Thus, by Theorem 2, the TP-cohomology of $\Omega$ is trivial in degree superior to zero just like in the book algebra example.

Furthermore, observe that $\Omega$ is orientable and that there is a 1 -form $\beta$ defining the foliation $\mathcal{F}$ such that $d \beta=0$, namely $\beta=d \theta$ where $\theta$ is given by

$$
e^{\imath \theta}=e^{-\varphi} x_{2}+\imath e^{\frac{-\varphi}{\tau}} x_{3} .
$$

Therefore, by Proposition 6 , the P-cohomology of $\Omega$ is the same as in the case of the book algebra:

$$
\begin{aligned}
& H_{\Lambda}^{0}(\Omega)=H_{\Lambda, \tan }^{0}(\Omega)=I(\Omega) \\
& H_{\Lambda}^{1}(\Omega) \simeq\{u d \theta: u \in I(\Omega)\} \simeq I(\Omega) \\
& H_{\Lambda}^{k}(\Omega) \simeq\{0\} \quad \forall k>1 .
\end{aligned}
$$

An analogous example is the Lie algebra defined by the brackets:

$$
\left[X_{1}, X_{2}\right]=X_{2}+X_{3}, \quad\left[X_{1}, X_{3}\right]=X_{3} .
$$

In this case, the 2-dimensional orbits can be parameterized by

$$
O_{\mu}=\left\{\left(s, e^{-t}\left(\mu_{2}-\mu_{3} t\right), e^{-t} \mu_{3}\right):(s, t) \in \mathbb{R}^{2}\right\},
$$

they are all cohomologically trivial and the regular Poisson manifold $(\Omega, \Lambda)$ is the set

$$
\Omega=\left\{\left(x_{1}, x_{2}, x_{3}\right): x_{2}^{2}+x_{3}^{2} \neq 0\right\}
$$

with its regular Poisson structure $\Lambda$. 
Now, let $G$ denote the following map

$$
G\left(t, x_{2}, x_{3}\right)=e^{-2 t}\left(\left(x_{2}-x_{3} t\right)^{2}+x_{3}^{2}\right)-1 .
$$

By the Implicit Function Theorem, the equation $G\left(t, x_{2}, x_{3}\right)=0$ determines $t$ implicitely as a smooth function $\psi: \mathbb{R}^{2} \backslash\{(0,0)\} \rightarrow \mathbb{R}$ of the variables $x_{2}, x_{3}$. The partial derivatives of $\psi$ are

$$
\begin{aligned}
\frac{\partial \psi}{\partial x_{2}} & =\frac{x_{2}-\psi x_{3}}{\left(x_{2}-\left(\psi-\frac{1}{2}\right) x_{3}\right)^{2}+\frac{3}{4} x_{3}^{2}} \\
\frac{\partial \psi}{\partial x_{3}} & =\frac{x_{3}+\psi^{2} x_{3}-\psi x_{2}}{\left(x_{2}-\left(\psi-\frac{1}{2}\right) x_{3}\right)^{2}+\frac{3}{4} x_{3}^{2}} .
\end{aligned}
$$

As before, the symplectic foliation $\mathcal{F}$ of $\Omega$ is given by a submersion, namely the map $\Pi: \Omega \rightarrow \mathbb{S}^{1}$ defined by

$$
\Pi\left(x_{1}, x_{2}, x_{3}\right)=\left(e^{-\psi\left(x_{2}, x_{3}\right)}\left(x_{2}-x_{3} \psi\left(x_{2}, x_{3}\right)\right), e^{-\psi\left(x_{2}, x_{3}\right)} x_{3}\right) .
$$

Thus, by Theorem 2, the TP-cohomology of $\Omega$ is trivial in degree superior to zero.

Moreover, $\Omega$ is orientable and there exists a 1 -form $\beta$ defining $\mathcal{F}$ such that $d \beta=0$, namely $\beta=d \theta$ where $\theta$ is now defined by

$$
e^{\imath \theta}=e^{-\psi}\left(\left(x_{2}-x_{3} \psi\right)+\imath x_{3}\right) .
$$

The P-cohomology of $\Omega$ is thus again:

$$
\begin{aligned}
H_{\Lambda}^{0}(\Omega) & =H_{\Lambda, \tan }^{0}(\Omega)=I(\Omega) \\
H_{\Lambda}^{1}(\Omega) & \simeq\{u d \theta: u \in I(\Omega)\} \\
& \simeq I(\Omega) \\
H_{\Lambda}^{k}(\Omega) & \simeq\{0\} \quad \forall k>1 .
\end{aligned}
$$

4.7. The simple Lie algebra $\mathfrak{s u}(2)$. This Lie algebra is defined by the following brackets:

$$
\left[X_{1}, X_{2}\right]=X_{3}, \quad\left[X_{2}, X_{3}\right]=X_{1}, \quad\left[X_{3}, X_{1}\right]=X_{2} .
$$

The nontrivial orbits are 2 -spheres and the regular Poisson manifold $(\Omega, \Lambda)$ is $\Omega=\mathfrak{s u}(2)^{*} \backslash\{0\}$ endowed with its regular Poisson structure $\Lambda$. We naturally identify $\Omega$ with $\mathbb{S}^{2} \times \mathbb{R}_{+}^{*}$. Using Theorem 3 , we get the TP-cohomology of $\Omega$ :

$$
\begin{aligned}
H_{\Lambda, \tan }^{0}(\Omega) & =I(\Omega)=C^{\infty}\left(\mathbb{R}_{+}^{*}\right) \\
H_{\Lambda, \tan }^{1}(\Omega) & =\{0\} \\
H_{\Lambda, \tan }^{2}(\Omega) & =\{[u \Lambda]: u \in I(\Omega)\} \\
& \simeq\left\{\left[u \omega_{\Lambda}\right]: u \in I(\Omega)\right\} \simeq I(\Omega)
\end{aligned}
$$


where $\omega_{\Lambda}$ denotes the foliated 2 -form associated to $\Lambda$ i.e.,

$$
\omega_{\Lambda}=\frac{x_{3} d x_{1} \wedge d x_{2}+x_{1} d x_{2} \wedge d x_{3}+x_{2} d x_{3} \wedge d x_{1}}{r^{2}} \quad\left(r=\sqrt{x_{1}^{2}+x_{2}^{2}+x_{3}^{2}}\right) .
$$

As for the other TP-cohomology spaces,

$$
H_{\Lambda, \tan }^{k}(\Omega)=\{0\} \quad \forall k>2 .
$$

It is important to note that, in this example, $\Omega$ is a product $S \times R$ like in Theorem 4. However, $\Lambda$ is not transversally constant so that Theorem 4 cannot be used.

We shall here compute the P-cohomology of $(\Omega, \Lambda)$. First, we have

$$
H_{\Lambda}^{0}(\Omega)=I(\Omega)=C^{\infty}\left(\mathbb{R}_{+}^{*}\right) .
$$

Now, note $\beta=d r$ and let $X$ be the Euler vector field $\left(X=\sum x_{i} \partial_{i}\right)$. Consider the tranversal distribution $\nu \mathcal{F}$ where, for each $x, \nu_{x}^{*} \mathcal{F}$ (resp. $\nu_{x} \mathcal{F}$ ) is spanned by $\beta_{x}$ (resp. $X_{x}$ ). Recall also that $H_{\Lambda}^{1}(\Omega)$ is reduced to the set:

$$
\left\{A \in \mathcal{V}_{1,0}(\Omega): \sigma^{\prime \prime}(A)=0 \text { and } \exists B \in \mathcal{V}_{0,1}(\Omega)\right.
$$

$$
\text { such that } \left.\sigma(A)+\sigma^{\prime \prime}(B)=0\right\} \text {. }
$$

It is easy to see that the set $\left\{A \in \mathcal{V}_{1,0}(\Omega): \sigma^{\prime \prime}(A)=0\right\}$ coincides with the set $\{u X: u \in I(\Omega)\}$. Now, since $\sigma(u X)=u \sigma(X)=u \Lambda$ for all $u$ in $I(\Omega)$ and that the class $[u \Lambda]$ in $H_{\Lambda, \tan }^{2}(\Omega)$ does not vanish unless $u=0$, we obtain

$$
H_{\Lambda}^{1}(\Omega)=\{0\} .
$$

Using Proposition 6 (i), we get

$$
H_{\Lambda}^{2}(\Omega) \simeq \frac{H_{\Lambda, \tan }^{2}(\Omega)}{\sigma\left(\mathcal{V}_{1,0}(\Omega)\right)} \oplus H^{1}\left(\Omega, \Phi^{1}(\mathcal{F})\right) .
$$

We saw that $\mathcal{V}_{1,0}(\Omega) \supset\{u X: u \in I(\Omega)\}$ and that $\sigma(u X)=u \Lambda$ for all $u$ in $I(\Omega)$. Moreover, by Proposition $6(\mathrm{ii}), H^{1}\left(\Omega, \Phi^{1}(\mathcal{F})\right)$ and $H_{\Lambda, \tan }^{1}(\Omega)$ are isomorphic. It follows that

$$
H_{\Lambda}^{2}(\Omega)=\{0\} .
$$

Finally, again by Proposition 6 , we have

$$
H_{\Lambda}^{3}(\Omega) \simeq H^{2}\left(\Omega, \Phi^{1}(\mathcal{F})\right) \text { and } H^{2}\left(\Omega, \Phi^{1}(\mathcal{F})\right) \simeq H_{\Lambda, \tan }^{2}(\Omega) .
$$

More explicitly,

$$
\begin{aligned}
H_{\Lambda}^{3}(\Omega) & =\{u[\Lambda \wedge X]: u \in I(\Omega)\} \\
& \simeq\left\{u\left[w_{\Lambda} \wedge d r\right]: u \in I(\Omega)\right\} \\
& \simeq I(\Omega) .
\end{aligned}
$$

Lastly,

$$
H_{\Lambda}^{k}(\Omega)=\{0\} \quad \forall k>3 .
$$


Remark 6. The Lie algebra $\mathfrak{s u}(2)$ provides an example of a regular Poisson manifold, namely $\Omega=\mathfrak{s u}(2)^{*} \backslash\{0\}$, which is exact $\left(\Lambda=\sigma(X)=\sigma\left(\sum x_{i} \partial_{i}\right)\right)$ without being tangentially exact $(\Lambda$ cannot be written in the form $\Lambda=\sigma(T)$ for any tangential vector field $T)$.

In fact, since for $\Omega=\mathfrak{s u}(2)^{*} \backslash\{0\}, H_{\Lambda}^{2}(\Omega)=\{0\}$ and $H_{\Lambda, \tan }^{2}(\Omega) \neq\{0\}$, the case of $\mathfrak{s u}(2)$ illustrates the fact that the TP-cohomology spaces are generally not imbedded in the corresponding $\mathrm{P}$-cohomology spaces, except in degree 1.

Note also that there is in $[\mathbf{X u}]$ a different and beautiful method to calculate the P-cohomology of $\mathfrak{s u}(2)^{*} \backslash\{0\}$ by means of symplectic groupoids. It consists of converting the P-cohomology to the de Rham cohomology of certain manifolds.

We propose now to discuss the remaining 3-dimensional Lie algebras. As we shall see in the sequel, all of them are pathological cases.

4.8. An interesting pathological example. Consider the Lie algebra $\mathfrak{h}$ given by the following brackets:

$$
\left[X_{1}, X_{2}\right]=X_{2}, \quad\left[X_{1}, X_{3}\right]=-X_{3}
$$

and denote by $H$ the connected and simply connected Lie group with Lie algebra $\mathfrak{h}$. For this Lie algebra, the 2-dimensional coadjoint orbits are the connected components of the hyperbolic cylinders $x_{2} x_{3}=$ const. and the half planes $x_{2}=0, \operatorname{sign}\left(x_{3}\right)$ fixed and $x_{3}=0, \operatorname{sign}\left(x_{2}\right)$ fixed. Each of them is cohomologically trivial and the regular Poisson manifold $(\Omega, \Lambda)$ associated to $\mathfrak{h}$ is the set

$$
\Omega=\left\{\left(x_{1}, x_{2}, x_{3}\right) \in \mathfrak{h}^{*}: x_{2}^{2}+x_{3}^{2} \neq 0\right\},
$$

endowed with its regular Poisson structure $\Lambda$. However, Theorem 2 cannot be applied because, as it was the case for $\mathfrak{g}_{4,1}$, the space of leaves $\Omega / H$ is not Hausdorff.

What is the TP-cohomology of $\Omega$ ?

As always, $H_{\Lambda, \tan }^{0}(\Omega)=I(\Omega)$. To describe $H_{\Lambda, \tan }^{1}(\Omega)$, we proceed as we did for $\mathfrak{g}_{4,1}$. We first observe that every tangential vector field $X$ is of the form $X=a \partial_{1}+b H_{x_{1}}$ with $a, b$ in $C^{\infty}(\Omega)$ and satisfies the equality $\sigma(X)=0$ if and only if $H_{x_{1}}(b)+\partial_{1}(a)=0$. Then, we fix a tangential vector field $X=a \partial_{1}+b H_{x_{1}}$ such that $\sigma(X)=0$. $X$ can be written in the form $X=\sigma f\left(f \in C^{\infty}(\Omega)\right)$ if and only if there exists $g(x)=g\left(x_{2}, x_{3}\right)$ in $C^{\infty}(\Omega)$ such that $H_{x_{1}}(g)(x)=a\left(0, x_{2}, x_{3}\right)$.

Consider now the change of variables: $u=x_{2} x_{3}, v=\frac{x_{2}^{2}-x_{3}^{2}}{2}$. It gives rise to a diffeomorphism $\varphi$ from $\{(u, v): u>0\}$ to $\left\{\left(x_{2}, x_{3}\right): x_{2}>0, x_{3}>0\right\}$ 
defined by

$$
\begin{aligned}
& \varphi(u, v)=\left(\sqrt{v+\sqrt{u^{2}+v^{2}}}, \frac{u}{\sqrt{v+\sqrt{u^{2}+v^{2}}}}\right) \quad \text { if } \quad v>0 \\
& \varphi(u, v)=\left(\frac{u}{\sqrt{-v+\sqrt{u^{2}+v^{2}}}}, \sqrt{-v+\sqrt{u^{2}+v^{2}}}\right) \quad \text { if } \quad v<0 \\
& \varphi(u, 0)=(\sqrt{u}, \sqrt{u}) .
\end{aligned}
$$

$\varphi$ can naturally be extended to $\{(u, v): u>0\} \cup\{(0, v): v \neq 0\}$. We still denote by $\varphi$ this natural extension.

The following result is, for $\mathfrak{h}$, the analog of Lemma 3 given in Section 3 for $\mathfrak{g}_{4,1}$.

Lemma 4. Let $a(x)=a\left(x_{2}, x_{3}\right)$ be in $C^{\infty}(\Omega)$. Suppose there exists some function $g(x)=g\left(x_{2}, x_{3}\right)$ in $C^{\infty}(\Omega)$ such that $H_{x_{1}}(g)=a$ and note $A=a \circ \varphi$, where $\varphi$ is as above. Then, the limit

$$
\lim _{\substack{u \rightarrow 0 \\ u>0}} \int_{-1}^{1} \frac{A(u, t)}{2 \sqrt{u^{2}+t^{2}}} d t
$$

exists.

Proof. By assumption, $H_{x_{1}}(g)=\left(x_{2} \partial_{2}-x_{3} \partial_{3}\right)(g)=a$. Changing variables, we get:

$$
2 \sqrt{u^{2}+v^{2}} \partial_{v}(G)=A
$$

where $G=g \circ \varphi$. Thus, on the set $\{(u, v): u>0\}, G$ is necessarily of the form

$$
G(u, v)=\int_{-1}^{v} \frac{A(u, t)}{2 \sqrt{u^{2}+t^{2}}} d t+\psi(u) .
$$

Moreover, since $\varphi(0,-1)=(0, \sqrt{2})$ and $g$ is continuous at $(0, \sqrt{2})$, $\lim _{\substack{u \rightarrow 0 \\ u>0}} G(u,-1)$ exists. Thus, $\lim _{\substack{u \rightarrow 0 \\ u>0}} \psi(u)$ exists too. In the same way, $\lim _{\substack{u \rightarrow 0 \\ u>0}} G(u, 1)$ exists and Lemma 3 is proved.

Now, using the vector fields $\widetilde{T_{\alpha}}=\widetilde{t_{\alpha}} \partial_{1}$ and $\widetilde{K_{\alpha}}=\widetilde{k_{\alpha}} \partial_{1}$ where

$$
\widetilde{t_{\alpha}}=\frac{1}{\left(x_{2}^{2}+x_{3}^{2}\right)^{\alpha}} \quad \text { and } \quad \widetilde{k_{\alpha}}=\frac{\exp \left(\frac{\alpha}{\left(x_{2}^{2}+x_{3}^{2}\right)^{2}}\right)}{\left(x_{2}^{2}+x_{3}^{2}\right)^{3}},
$$

one can see that the space $H_{\Lambda, \tan }^{1}(\Omega)$ is infinite dimensional.

As for the other TP-cohomology spaces,

$$
H_{\Lambda, \tan }^{k}(\Omega)=\{0\} \quad \forall k>1 .
$$


To compute the P-cohomology of $\Omega$, let us say that the symplectic foliation $\mathcal{F}$ of $\Omega$ is orientable and is defined by the nonsingular 1 -form $\beta=x_{2} d x_{3}+$ $x_{3} d x_{2}(d \beta=0)$. By Proposition 6 and if we note $X=\frac{x_{2} \partial_{3}+x_{3} \partial_{2}}{x_{2}^{2}+x_{3}^{2}}$, we have

$$
\begin{aligned}
& H_{\Lambda}^{0}(\Omega)=I(\Omega) \\
& H_{\Lambda}^{1}(\Omega)=H_{\Lambda, \tan }^{1}(\Omega) \oplus\{u X: u \in I(\Omega)\} \\
& H_{\Lambda}^{2}(\Omega) \simeq H^{1}\left(\Omega, \Phi^{1}(\mathcal{F})\right) \simeq H_{\Lambda, \tan }^{1}(\Omega) \\
& H_{\Lambda}^{3}(\Omega) \simeq H^{2}\left(\Omega, \Phi^{1}(\mathcal{F})\right) \simeq H_{\Lambda, \tan }^{2}(\Omega)=\{0\} \\
& H_{\Lambda}^{k}(\Omega)=\{0\} \quad \forall k>3 .
\end{aligned}
$$

Thus, the large space $H_{\Lambda, \tan }^{1}(\Omega)$ happens in the P-cohomology. In other words, the spaces $H_{\Lambda}^{1}(\Omega)$ and $H_{\Lambda}^{2}(\Omega)$ are infinite dimensional.

Remark 7. Consider the family of 3-dimensional Lie algebras given by:

$$
\left[X_{1}, X_{2}\right]=X_{2}, \quad\left[X_{1}, X_{3}\right]=\frac{1}{\tau} X_{3} \quad \tau<-1 .
$$

One can prove that the TP-cohomology of $\Omega$ in these examples is essentially the same as in the case of $\mathfrak{h}$ because of a similar leaf structure. The situation seems more complicated for the P-cohomology since Proposition 6 cannot be used.

4.9. The case of $\mathfrak{s l}(2)$. We want now to show how the TP-cohomology computation for $\mathfrak{s l}(2)$ leads to the same conclusions as in the case of $\mathfrak{g}_{4,1}$ or $\mathfrak{h}$.

The Lie algebra $\mathfrak{s l}(2)$ is given by the brackets:

$$
\left[X_{1}, X_{2}\right]=-X_{3}, \quad\left[X_{3}, X_{1}\right]=X_{2}, \quad\left[X_{3}, X_{2}\right]=-X_{1} .
$$

Let us identify $\mathfrak{s l}(2)$ with its dual $\mathfrak{s l}(2)^{*}$ by means of the Killing form. Then the orbit decomposition is as follows. There are three orbits in the light cone:

$$
\begin{aligned}
& W_{0}=\{0\} ; \\
& W_{+}=\left\{\left(\mu_{1}, \mu_{2}, \mu_{3}\right): \mu_{1}^{2}+\mu_{2}^{2}-\mu_{3}^{2}=0, \mu_{3}>0\right\} ; \\
& W_{-}=\left\{\left(\mu_{1}, \mu_{2}, \mu_{3}\right): \mu_{1}^{2}+\mu_{2}^{2}-\mu_{3}^{2}=0, \mu_{3}<0\right\} .
\end{aligned}
$$

Moreover, the hyperbolic orbits are single sheeted hyperboloids outside the cone:

$$
W_{k}=\left\{\left(\mu_{1}, \mu_{2}, \mu_{3}\right): \mu_{1}^{2}+\mu_{2}^{2}-\mu_{3}^{2}=k^{2}\right\} \quad(k>0)
$$

and double sheeted hyperboloids inside the cone (each of the sheets being a different orbit):

$$
W_{h}=\left\{\left(\mu_{1}, \mu_{2}, \mu_{3}\right): \mu_{1}^{2}+\mu_{2}^{2}-\mu_{3}^{2}=-h^{2}, \mu_{3} h>0\right\} \quad(h \neq 0) .
$$


The regular Poisson manifold $(\Omega, \Lambda)$ associated to $\mathfrak{s l}(2)$ is thus the set

$$
\Omega=\left\{\left(x_{1}, x_{2}, x_{3}\right) \in \mathfrak{s l}(2)^{*}: x_{1}^{2}+x_{2}^{2}+x_{3}^{2} \neq 0\right\},
$$

with its regular Poisson structure $\Lambda$ and the space of leaves is clearly not Hausdorff.

As always, $H_{\Lambda, \tan }^{0}(\Omega)=I(\Omega)$.

How about $H_{\Lambda, \tan }^{1}(\Omega)$ ? Assume first that $X$ is a tangential vector field of the form

$$
X=a H_{x_{3}}
$$

where $a$ is in $C^{\infty}(\Omega)$. One can easily check that $X=\sigma f$ for a function $f$ in $C^{\infty}(\Omega)$ if and only if $f$ satisfies the following properties:

$$
\begin{aligned}
& H_{x_{1}}(f)=x_{2} a \\
& H_{x_{2}}(f)=-x_{1} a \\
& H_{x_{3}}(f)=0 .
\end{aligned}
$$

Consider the open set $U=\left\{\left(x_{1}, x_{2}, x_{3}\right) \in \Omega: x_{1}^{2}+x_{2}^{2}-x_{3}^{2}>0\right\}$ which can be parameterized by:

$$
\begin{aligned}
& x_{1}=-p \sin (q)+z \cos (q) \\
& x_{2}=-p \cos (q)-z \sin (q) \\
& x_{3}=p .
\end{aligned}
$$

Using this parameterization, we see that, if $X=\sigma f$, then $\partial_{p}(f)=-a$ and $\partial_{q}(f)=0$. In other words, if $X=\sigma f$, then $f_{\mid U}$ must depend only on $(p, z)$ and be of the form

$$
f=f(p, z)=-\int_{-1}^{p} a(s, q, z) d s+\psi(z) .
$$

Consider the vector fields $\hat{T}_{\alpha}$ ans $\hat{K}_{\alpha}, \alpha>\frac{1}{2}$, defined by

$$
\hat{T_{\alpha}}=\hat{t_{\alpha}} H_{x_{3}} \quad \text { and } \quad \hat{K}_{\alpha}=\hat{k_{\alpha}} H_{x_{3}}
$$

where

$$
\hat{t_{\alpha}}=\frac{1}{\left(x_{1}^{2}+x_{2}^{2}+x_{3}^{2}\right)^{\alpha}} \quad \text { and } \quad \hat{k_{\alpha}}=\frac{\exp \left(\frac{\alpha}{x_{1}^{2}+x_{2}^{2}+x_{3}^{2}}\right)}{\left(x_{1}^{2}+x_{2}^{2}+x_{3}^{2}\right)^{2}} .
$$

Then, with the same arguments as in the examples of $\mathfrak{g}_{4,1}$ and $\mathfrak{h}$, one can prove that the space $H_{\Lambda, \tan }^{1}(\Omega)$ is infinite dimensional.

The same is true for $H_{\Lambda, \tan }^{2}(\Omega)$. Indeed, take a tangential 2-tensor field $A$. Necessarily, there is a function $\varphi$ in $C^{\infty}(\Omega)$ such that

$$
A=\varphi \Lambda=\varphi\left(x_{3} \partial_{2} \wedge \partial_{1}+x_{2} \partial_{3} \wedge \partial_{1}+x_{1} \partial_{2} \wedge \partial_{3}\right)
$$


and $A$ satisfies $\sigma(A)=0$. Now, suppose there exists a tangential vector field $B$ such that $A=\sigma(B)$. If we note $B=a H_{x_{1}}+b H_{x_{2}}+c H_{x_{3}}$ where $a, b, c$ are in $C^{\infty}(\Omega)$, then we obtain

$$
\text { (\#) } \quad H_{x_{1}}(a)+H_{x_{2}}(b)+H_{x_{3}}(c)=\varphi .
$$

Translating (\#) on the open set $U=\left\{\left(x_{1}, x_{2}, x_{3}\right) \in \Omega: x_{1}^{2}+x_{2}^{2}-x_{3}^{2}>0\right\}$, we get

$$
\left(-x_{2} \partial_{p}-\sin (q) \partial_{q}\right)(a)+\left(x_{1} \partial_{p}-\cos (q) \partial_{q}\right)(b)+\partial_{q}(c)=\varphi .
$$

Let us integrate the above expression between 0 and $2 \pi$ as follows

$$
\begin{aligned}
& p \partial_{p}\left(\int_{0}^{2 \pi}(\cos (q) a-\sin (q) b) d q\right)+z \partial_{p}\left(\int_{0}^{2 \pi}(\sin (q) a+\cos (q) b) d q\right) \\
& \quad+\int_{0}^{2 \pi}\left(-\sin (q) \partial_{q}(a)-\cos (q) \partial_{q}(b)\right) d q \\
& =p \partial_{p}\left(\int_{0}^{2 \pi}(\cos (q) a-\sin (q) b) d q\right)+z \partial_{p}\left(\int_{0}^{2 \pi}(\sin (q) a+\cos (q) b) d q\right) \\
& \quad+\int_{0}^{2 \pi}(\cos (q) a-\sin (q) b) d q \\
& =\int_{0}^{2 \pi} \varphi(p, q, z) d q .
\end{aligned}
$$

Assume that $\varphi$ depends only on the variables $p, z$. Moreover, define the functions $H$ and $g$ by

$$
H(p, z)=\int_{0}^{2 \pi}(\cos (q) a-\sin (q) b) d q, \quad g(p, z)=\int_{0}^{2 \pi}(\sin (q) a+\cos (q) b) d q .
$$

Then we have

$$
p \partial_{p} H+H=2 \pi \varphi-z \partial_{p}(g) .
$$

Thus, on the open set $U \cap\{p>0\}, H$ is necessarily of the form

$$
H=\frac{1}{p}\left(\int_{1}^{p} 2 \pi \varphi(s, z) d s-z g(p, z)+z g(1, z)+\psi_{1}(z)\right) .
$$

Similarly, on the open set $U \cap\{p<0\}, H$ is necessarily of the form

$$
H=\frac{1}{p}\left(\int_{-1}^{p} 2 \pi \varphi(s, z) d s-z g(p, z)+z g(-1, z)+\psi_{2}(z)\right) .
$$

The function $H(p, z)$ can be extended at the points $(p, 0)$ with $p \neq 0$, therefore $\psi_{1}(z)$ et $\psi_{2}(z)$ have a limit when $z(>0)$ tends to zero. Moreover, $H(p, z)$ can also be extended at the points $(0, z)$ with $z \neq 0$. Thus, for all $z \neq 0$, we get

$$
\int_{1}^{0}(2 \pi \varphi(s, z)) d s-z g(0, z)+z g(1, z)+\psi_{1}(z)=0
$$


and

$$
\int_{-1}^{0}(2 \pi \varphi(s, z)) d s-z g(0, z)+z g(-1, z)+\psi_{2}(z)=0
$$

It follows that

$$
\int_{-1}^{1}(2 \pi \varphi(s, z)) d s-z g(1, z)+z g(-1, z)-\psi_{1}(z)+\psi_{2}(z)=0 .
$$

In other words, if $A=\varphi \Lambda$ where $\varphi_{\mid U}=\varphi(p, z)$ and if there exists a tangential vector field $B$ such that $A=\sigma(B)$, then the limit

$$
\lim _{\substack{z \rightarrow 0 \\ z>0}} \int_{-1}^{1} 2 \pi \varphi(s, z) d s
$$

should exist. Now to see that the space $H_{\Lambda, \tan }^{2}(\Omega)$ is infinite dimensional, one can take the vector fields $\hat{t_{\alpha}} \Lambda$ where $\hat{t_{\alpha}}=\frac{1}{\left(x_{1}^{2}+x_{2}^{2}+x_{3}^{2}\right)^{\alpha}}$ and $\hat{k_{\alpha}} \Lambda$ where $\hat{k_{\alpha}}=\frac{\exp \left(\frac{\alpha}{x_{1}^{2}+x_{2}^{2}+x_{3}^{2}}\right)}{\left(x_{1}^{2}+x_{2}^{2}+x_{3}^{2}\right)^{2}}$.

Lastly,

$$
H_{\Lambda, \tan }^{k}(\Omega)=0 \quad \forall k>2 .
$$

Let us also say that the symplectic foliation $\mathcal{F}$ of $\Omega$ is orientable and is defined by the nonsingular 1 -form $\beta=x_{1} d x_{1}+x_{2} d x_{2}-x_{3} d x_{3}$, which satisfies $d \beta=0$. Thus, using Proposition 6 , one can see that all the P-cohomology spaces $H_{\Lambda}^{k}(\Omega)(1 \leq k \leq 3)$ are infinite dimensional.

We finish with an application of our cohomology calculations for the classification of tangential star products:

Remark 8. As we just proved in this section, the second TP-cohomology space of $\Omega$ is zero for any 3-dimensional Lie algebra except for $\mathfrak{s u}(2)$ and $\mathfrak{s l}(2)$. This implies the uniqueness (up to equivalence) of the tangential star products on $\Omega$ for any non-simple 3-dimensional Lie algebra.

\section{Concluding remarks.}

As shown in Section 2, for a regular Poisson manifold, the TP-cohomology coincides with the leafwise de Rham cohomology of the symplectic foliation, thus unlike the P-cohomology, does not depend on the symplectic structure along the leaves. That relates the task of computing the TPcohomology of regular Poisson manifolds to nontrivial questions of foliation theory. Roughly speaking, the TP-cohomology not only contains the de Rham cohomology of the leaves, but also translates the foliation complexity which includes essentially the relative position of the leaves. As seen from the computations of Sections 3 and 4, when the space of leaves is not Hausdorff, this TP-cohomology is very large and hardly describable. To finish, 
let us say that the TP-cohomology spaces are involved in the P-cohomology, for instance we have the inclusion $H_{\Lambda, \tan }^{1}(M) \subset H_{\Lambda}^{1}(M)$ for any regular Poisson manifold $M$. Thus, our calculations and comments can be of some help to understand why the P-cohomology itself is, as often said, so difficult to compute.

Acknowledgements. I am infinitely grateful to my advisor Didier ARNAL for his continual and helpful guidance throughout this work. I also thank the referee for illuminating comments and pointing out a mistake in the first version.

\section{References}

[ACG] D. Arnal, M. Cahen and S. Gutt, Deformations on coadjoint orbits, J. Geom. Phys., 3(3) (1986), 327-351, MR 88k:58047, Zbl 0616.58013.

[APP] J.A. de Azcarraga, A.M. Perelomov and J.C. Perez Bueno, The Schouten Nijenhuis bracket, cohomology and generalized Poisson structures, J. Phys. A.: Math. Gen., 29 (1996), 7993-8009, MR 98h:58058, Zbl 0898.58026.

[BLM] J. Boidol, J. Ludwig and D. Müller, On infinitely small orbits, Studia Mathematica, LXXXVIII (1988), MR 89f:22009, Zbl 0662.22006.

[Bon] P. Bonnet, Paramétrisation du dual d'une algèbre de Lie nilpotente, Ann. Inst. Fourier Grenoble, 38(3) (1988), 169-197, MR 90d:22008, Zbl 0618.22004.

[Br] R. Bryant, An introduction to Lie groups and symplectic geometry, D.S. Freed (ed.) et al., Geometry and quantum field theory; Lecture notes from the graduate summer school program, June 22-July 20, 1991, Park City, UT, USA; Providence, RI, American Mathematical Society; IAS/Park City Math. Ser., 1 (1995), 5-181, MR 96i:58002, Zbl 0853.53024.

[Bry] J.L. Brylinski, Loop Spaces, Characteristic Classes and Geometric Quantization, Progress in Mathematics, 107, Birkhäuser, Boston, 1993, MR 94b:57030, Zbl 0823.55002 .

[CGR] M. Cahen, S. Gutt and J. Rawnsley, On tangential star products for the coadjoint Poisson structure, Comm. Math. Phys., 180 (1996), 99-108, MR 97e:58101, Zbl 0859.17017.

[CW] A. Cannas Da Silva and A. Weinstein, Geometric Models for Noncommutative Algebras, Berkeley Mathematics Lecture Notes, 10, American Mathematical Society, 1999, MR 2001m:58013.

[CG] L. Corwin and F.P. Greenleaf, Representations of nilpotent Lie groups and their applications, Part 1: Basic theory and examples, Cambridge studies in advanced mathematics, 1990, MR 92b:22007, Zbl 0704.22007.

[DH] P. Dazord et G. Hector, Intégration Symplectique des variétés de Poisson totalement asphériques, in 'Symplectic Geometry, Groupoids and Integrable Systems', Séminaire Sud Rhodanien de Géométrie à Berkeley, 1989, (P. Dazord and A. Weinstein, eds.); MSRI Publ., 20, Springer-Verlag, Berlin-Heidelberg-New York, (1991), 37-72, MR 92k:58281, Zbl 0735.58003.

[Die] J. Dieudonné, Eléments d'analyse, Cahiers scientifiques, XXXIII(3), GauthierVillars, 1974, MR 42 \#5266, Zbl 0208.31802. 
[GK] M. Goze and Y. Khakimdjanov, Nilpotent Lie algebras, Mathematics and its Applications, 361, Kluwer Academic Publishers Group, 1996, MR 97e:17017, Zbl 0845.17012.

[Gré] G. Grélaud, Sur les représentations des groupes de Lie résolubles, Thèse, Université de Poitiers, 1984.

[Hue] J. Huebschmann, Poisson cohomology and quantization, J. für Reine und Angew. Math., 408 (1990), 57-113, MR 92e:17027, Zbl 0699.53037.

[Kon] M. Kontsevich, Deformation quantization of Poisson manifolds, preprint qalg/9709040, 1997.

[Kos] J.L. Koszul, Crochet de Schouten-Nijenhuis et cohomologie, Elie Cartan et les Mathématiques d'aujourd'hui, Astérisque hors série, Société Math. de France, (1985), 257-271, MR 88m:17013, Zbl 0615.58029.

[Li1] A. Lichnerowicz, Les variétés de Poisson et leurs algèbres de Lie associées, J. Diff. Geom., 12 (1977), 253-300, MR 58 \#18565, Zbl 0405.53024.

[Li2] Variétés de Poisson et feuilletages, Ann. Fac. Sci. Toulouse Math, 4 (1982), 195-262, MR 84m:58042, Zbl 0517.58029.

[Mas] M. Masmoudi, Tangential formal deformations of the Poisson bracket and tangential star products on a regular Poisson manifold, J. Geom. Phys., 9 (1992), 155-171, MR 94a:58216, Zbl 0765.53023.

[Pe1] N.V. Pedersen, Geometric quantization and the universal enveloping algebra of a nilpotent Lie group, Trans. A. M. S., 315(2) (1989), 511-563, MR 90c:22026, Zbl 0684.22004 .

[Pe2] Geometric quantization and nilpotent Lie groups, A collection of examples, University of Copenhagen Denmark, (1988), 1-180.

[Puk] L. Pukanszky, Leçon sur les Représentations des Groupes, Monographies de la Société Mathématique de France, 2, Dunod, Paris, 1967, MR 36 \#311, Zbl 0152.01201.

[SG] M. Saint-Germain, Poisson algebras and transverse structures, J. Geom. Phys., 31 (1999), 153-194, MR 2000m:53117, Zbl 0958.17006.

[Va1] I. Vaisman, Remarks on the Lichnerowicz-Poisson cohomology, Ann. Inst. Fourier Grenoble, 40 (1990), 951-963, MR 92c:58155, Zbl 0708.58010.

[Va2] Lectures on the Geometry of Poisson Manifolds, Progress in Mathematics, 118, Birkhäuser, Basel, 1994, MR 95h:58057, Zbl 0810.53019.

[Ver] M. Vergne, La structure de Poisson sur l'algèbre symétrique d'une algèbre de Lie nilpotente, Bull. Soc. Math. Fr., 100 (1972), 301-335, MR 52 \#657, Zbl 0256.17002.

[Wei] A. Weinstein, The local structure of Poisson manifolds, J. Diff. Geom., 18 (1983), 523-557, MR 87k:58099, Zbl 0524.58011.

[Xu] P. Xu, Poisson cohomology of regular Poisson manifolds, Ann. Inst. Fourier Grenoble, 42 (1992), 967-988, MR 94d:58167, Zbl 0759.58020.

Received May 19, 2000 and revised February 16, 2001.

DÉPARTEMENT DE MAThÉMATIQUES

UPRES-A CNRS 7035

UNIVERsité DE METZ

Ile DU SAUlCy

57045 Metz Cedex 01, France 
E-mail address: gammella@poncelet.univ-metz.fr 